\title{
The potential of functional near-infrared spectroscopy-based neurofeedback - a systematic review and recommendations for best practice
}

\author{
Simon H. Kohl ${ }^{1,2}$, David M.A. Mehler ${ }^{3}$, Michael Lührs ${ }^{4,5}$, Robert T. Thibault ${ }^{6,7}$, Kerstin \\ Konrad $^{1,2}$, Bettina Sorger ${ }^{5}$ \\ ${ }^{1}$ JARA-Institute Molecular Neuroscience and Neuroimaging (INM-11), Jülich Research Centre, \\ Jülich, Germany
}

${ }^{2}$ Child Neuropsychology Section, Department of Child and Adolescent Psychiatry, Psychosomatics and Psychotherapy, Medical Faculty, RWTH Aachen University, Germany

${ }^{3}$ Department of Psychiatry, University of Münster, Münster, Germany

${ }^{4}$ Brain Innovation B.V., Research Department, Maastricht, Netherlands

${ }^{5}$ Faculty of Psychology and Neuroscience, Department of Cognitive Neuroscience, Maastricht University, Netherlands

${ }^{6}$ School of Psychological Science, University of Bristol, Bristol, United Kingdom

${ }^{7}$ MRC Integrative Epidemiology Unit at the University of Bristol, Bristol, United Kingdom

* Correspondence:

Simon H. Kohl

si.kohl@fz-juelich.de, $\underline{\text { simon.h.kohl@gmail.com }}$

Keywords: Real-time data analysis 1 , functional near-infrared spectroscopy, neurofeedback3, systematic review4, clinical translation, self-regulation, $_{6}$, brain-computer interfacing 7 


\begin{abstract}
Background: The effects of electroencephalography (EEG) and functional magnetic resonance imaging (fMRI)-neurofeedback on brain activation and behaviors have been studied extensively in the past. More recently, researchers have begun to investigate the effects of functional near-infrared spectroscopy-based neurofeedback (fNIRS-neurofeedback). FNIRS is a functional neuroimaging technique based on brain hemodynamics, which is easy to use, portable, inexpensive, and has reduced sensitivity to movement artifacts.
\end{abstract}

Method: We provide the first systematic review and database of fNIRS-neurofeedback studies, synthesizing findings from 22 peer-reviewed studies (including a total of N=441 participants; 337 healthy, 104 patients). We (1) give a comprehensive overview of how fNIRS-neurofeedback training protocols were implemented, (2) review the online signal-processing methods used, (3) evaluate the quality of studies using pre-set methodological and reporting quality criteria and also present statistical sensitivity/power analyses, (4) investigate the effectiveness of fNIRS-neurofeedback in modulating brain activation, and (5) review its effectiveness in changing behavior in healthy and pathological populations.

Results and discussion: (1-2) Published studies are heterogeneous (e.g., neurofeedback targets, investigated populations, applied training protocols, and methods). (3) Large randomized controlled trials are still lacking. In view of the novelty of the field, the quality of the published studies is moderate. We identified room for improvement in reporting important information and statistical power to detect realistic effects. (4) Several studies show that people can regulate hemodynamic signals from cortical brain regions with fNIRS-neurofeedback and (5) these studies indicate the feasibility of modulating motor control and prefrontal brain functioning in healthy participants and ameliorating symptoms in clinical populations (stroke, ADHD, autism, and social anxiety). However, valid conclusions about specificity or potential clinical utility are premature.

Conclusion: Due to the advantages of practicability and relatively low cost, fNIRS-neurofeedback might provide a suitable and powerful alternative to EEG and fMRI neurofeedback and has great potential for clinical translation of neurofeedback. Together with more rigorous research and reporting practices, further methodological improvements may lead to a more solid understanding of fNIRS-neurofeedback. Future research will benefit from exploiting the advantages of fNIRS, which offers unique opportunities for neurofeedback research. 
fNIRS-Neurofeedback - a systematic review

\section{Introduction}

Functional near-infrared spectroscopy (fNIRS) is a growing functional neuroimaging technique that exploits the principles of near-infrared (NIR) spectroscopy and brain hemodynamics. Human tissues, including brain tissue, are relatively transparent to light in the NIR range (650-1000 nm). If NIR light is directed onto the surface of the head most of the light scatters within the underlying tissue, while some of the light is absorbed by pigmented compounds (chromophores). The main chromophore hemoglobin (red blood cells transporting oxygen) absorbs and attenuates the NIR light, and the absorption spectrum of hemoglobin is dependent on the oxygenation level, i.e. oxy-(HbO) $>800 \mathrm{~nm}$ and deoxyhemoglobin $(\mathrm{HbR})<800 \mathrm{~nm}$. This principle is utilized by fNIRS to detect relative changes of $\mathrm{HbO}$ and $\mathrm{HbR}$ levels and thereby indirectly estimating brain activation in the underlying brain tissue via optical sensors placed on the surface of the head (see Ferrari and Quaresima, 2012 and Pinti et al., 2018b).

Compared to other neuroimaging modalities, such as functional magnetic resonance imaging (fMRI), electroencephalography (EEG), and magnetoencephalography (MEG), fNIRS shows both advantages and disadvantages. First, the spatial and temporal resolution of fNIRS lies between fMRI and EEG. It provides higher spatial resolution (between $2-3 \mathrm{~cm}$ ) than EEG and potentially higher temporal resolution than fMRI, due to a higher sampling rate. The depth of fNIRS measurements is restricted to neocortical brain regions (Pinti et al., 2018b). FNIRS also has a lower spatial resolution and lower signal-to-noise ratio compared to fMRI (Cui et al., 2011). However, the practicability of fNIRS is a major advantage over fMRI: it is easier to use, portable, safe, nearly silent, inexpensive, and requires little setup time. Moreover, fNIRS measurements tolerate more head motion compared to EEG and fMRI measurements. This makes it possible to use fNIRS in more naturalistic

environments/situations (e.g., allowing neural activity to be recorded during overt speech, movement, and direct interaction with another person). Furthermore, it permits populations to be investigated that are more likely to show head motion (e.g., neurological or psychiatric patients or infants) and situations that do not allow fMRI measurements (e.g., participants with ferromagnetic implants or claustrophobia). For recent reviews on the use of fNIRS in neuroscience see (Pinti et al., 2018b; Quaresima and Ferrari, 2019).

Given the advantages of fNIRS over other neuroimaging modalities, this technique has been increasingly used as a tool for neurofeedback (Ehlis et al., 2018). During neurofeedback training, participants are trained to self-regulate their brain activity, generally with the ultimate goal of changing behavior or cognitive/emotional functions (for reviews see Paret et al., 2019; Sitaram et al., 2017; Thibault et al., 2016). Figure 0.2 (upper part) shows a typical fNIRS-neurofeedback setup. Changes in $\mathrm{HbO}, \mathrm{HbR}$, or total hemoglobin $(\mathrm{tHb})$ are assessed via optodes placed on the participants' heads covering a certain brain region of interest and are usually fed back to the subject in the form of visual representations. Individuals can then use this feedback information to learn successful selfregulation of brain activity and ideally transfer this skill to daily life. Successful neurofeedback training usually requires several neurofeedback sessions (1-5 sessions for fMRI-neurofeedback and up to 30 sessions for EEG-neurofeedback (see Thibault et al., 2016)), which is costly and difficult to perform with fMRI.

To date, there has been no comprehensive systematic review of fNIRS-neurofeedback studies. The available reviews are either not systematic or are selective (not covering all published fNIRSneurofeedback work). Some reviews focus more on general aspects of fNIRS-based brain-computer interfacing (e.g., Ehlis et al., 2018; Naseer and Hong, 2015; Thibault et al., 2016). For example, a recent review by Ehlis et al. (2018) reviewed several of their own studies alongside a few other 
experiments. They concluded that fNIRS-neurofeedback training can enable participants to regulate their hemodynamic responses deliberately and that this training may induce changes in brain functions over time. Further, Ehlis et al. (2018) conclude that if future studies confirmed initial findings, fNIRS-neurofeedback may become a complementary or even alternative treatment option for neuropsychiatric disorders.

The present systematic review is divided into five stand-alone sections. We (1) synthesize information about training protocols; (2) provide an overview of the methods used for online signalprocessing to calculate the feedback signal; (3) critically evaluate the quality of published studies including experimental designs, reporting (Ros et al., 2020; Tufanaru et al., 2017), and statistical power; (4) assess and discuss the effectiveness of fNIRS-neurofeedback to regulate and induce prepost changes in brain activity; and (5) assess and discuss its effectiveness in inducing changes in behavioral/cognitive/emotional ${ }^{1}$ outcome measurements in healthy and pathological populations and also review the clinical potential of fNIRS-neurofeedback. We finish the review with a discussion arising from the findings of the five sections and also touch on the future of fNIRS-neurofeedback research.

${ }^{1}$ To simplify the text, in the following we subsume the conceptually different behavioral, cognitive, and emotional outcome variables under the general term behavior. 
fNIRS-Neurofeedback - a systematic review

\section{Methods}

The study protocol for this systematic review was registered on PROSPERO and can be accessed at https://www.crd.york.ac.uk/PROSPERO/display_record.php?RecordID=141049. Data from this systematic review are available at the Open Science Framework (see https://osf.io/hnxfq/). We followed the Preferred Reporting Items for Systematic Reviews and Meta-Analyses (PRISMA) guidelines (Moher et al., 2009).

\section{Search strategy}

We searched the following electronic bibliographic databases for studies published up until 3 July 2019: PubMed/MEDLINE, Web of Knowledge/Web of Science, Scopus, and EMBASE. Additional searches were conducted using the Real-time Functional Imaging and Neurofeedback (rtFIN) database (rtfin.org), Cochrane Reviews library database (cochranelibrary.com), Clinicaltrials.gov, scholar.google.de, and preprint servers: biorxiv.org, arxiv.org, psyarxiv.com, medrxiv.org and osf.io. The following search terms were used: ("functional near infrared spectroscopy" OR "fNIRS" OR "near infrared spectroscopy" OR "NIRS") AND ("real-time" OR "real time" OR "neurofeedback" OR "biofeedback" OR "Brain-Computer Interface" OR "Brain computer interface" OR "BCI" OR "Brain-Machine Interface" OR "Brain Machine Interface" OR "BMI"). More details of the search strategy can be found in the protocol or via the following link: https://www.crd.york.ac.uk/PROSPEROFILES/141049_STRATEGY_20190703.pdf

\section{Study selection}

We included all published articles with study designs that applied fNIRS-neurofeedback training to regulate brain activity and/or behavior in healthy or patient populations. The field is very young and to date no randomized controlled trials have been published. Hence, we applied rather loose inclusion criteria, also including non-controlled, pilot, feasibility, and proof-of-concept studies involving at least four participants. Studies applying fNIRS only for the purpose of brain-based communication or control of devices were excluded. After removing duplicates, the titles and abstracts of 2821 articles were screened and the full-text of the remaining 33 articles was retrieved and assessed for eligibility. 22 studies met the inclusion criteria and were considered in the qualitative synthesis (see Figure 0.1), involving a total of 441 participants (337 healthy participants and 104 patients). 


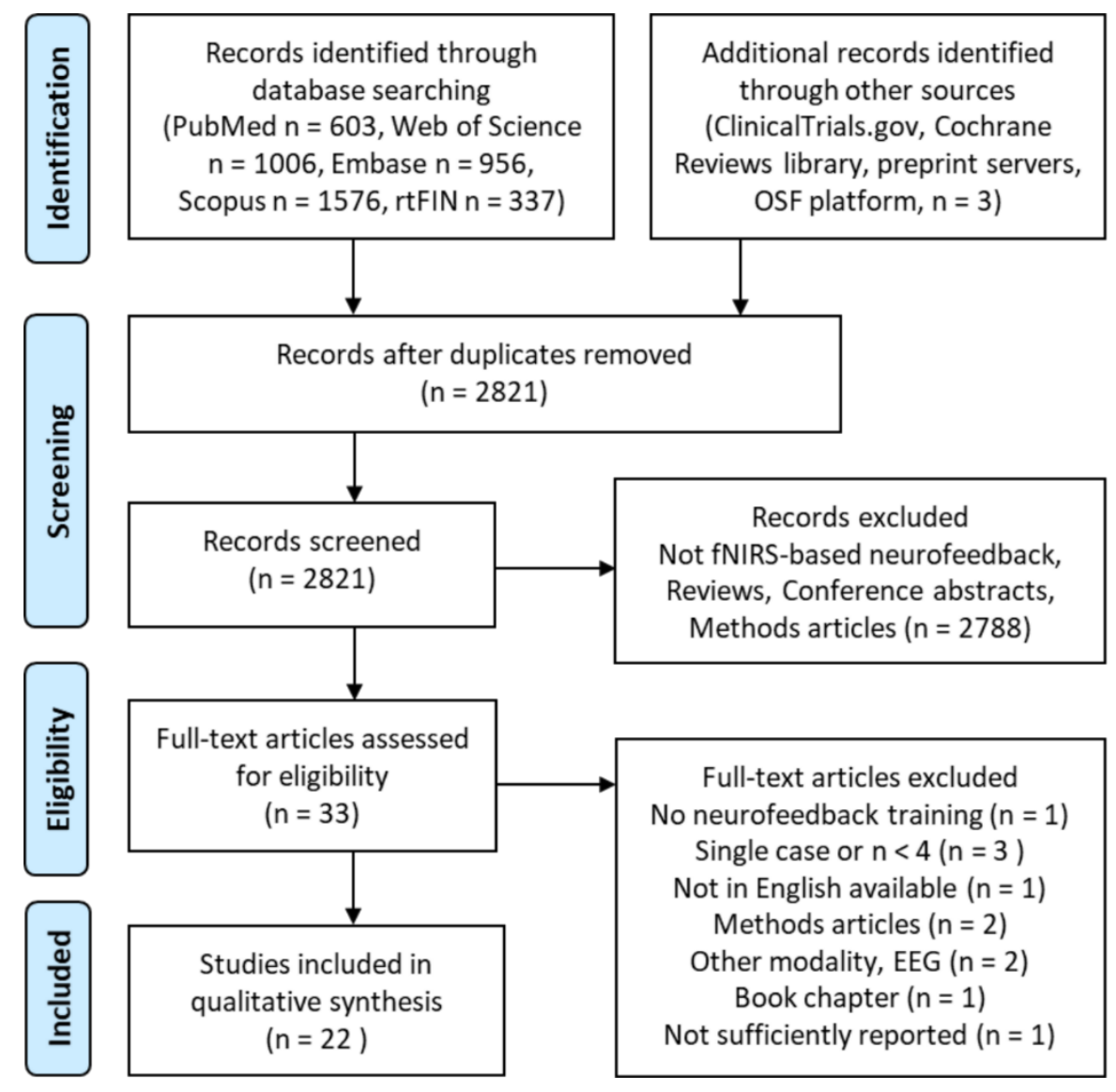

Figure 0.1. Search decision flow diagram according to Preferred Reporting Items for Systematic Reviews and Meta-Analyses (PRISMA; Moher et al., 2009)

\section{Data extraction and analysis}

A spreadsheet was used to document data extracted from the studies. Extracted data included: information about study population and study design, details of the neurofeedback protocol and control conditions; methods used for online signal-processing to calculate the feedback information, and outcomes of the neurofeedback training categorized into behavioral and neural effects within and independent of targeted brain regions (see supplementary file). After finishing the data extraction, the spreadsheet was sent to all corresponding authors of the included studies to ask for corrections. 15 of 22 authors replied and either approved the data extraction or sent minor corrections. We also gave authors the opportunity to comment on a preprint version of the manuscript, which was uploaded at the Open Science Framework (see https://osf.io/hnxfq/) before submission. Further methodological details will be provided in the respective sections. 


\section{Results and discussion}

We present and discuss the results of this systematic review in five sections (depicted in Figure 0.2).

1) Training protocols

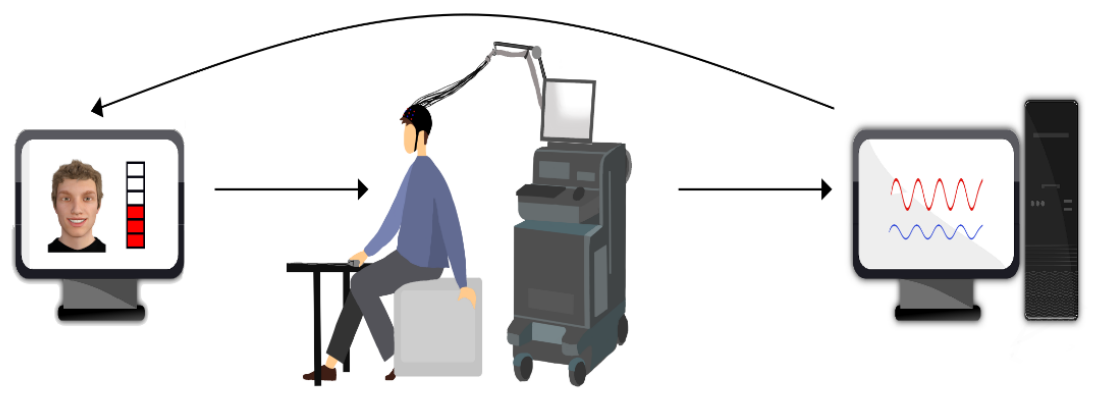

3) Quality of published studies

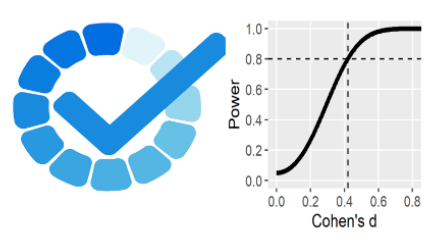

4) Neural effects of fNIRS-neurofeedback

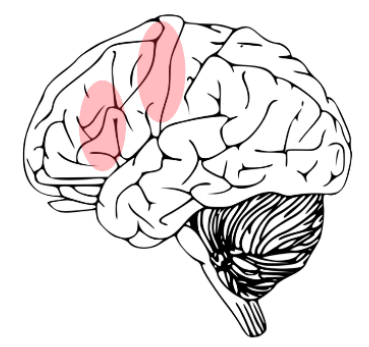

5) Behavioral effects in healthy and clinical populations

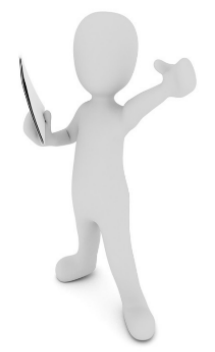

Figure 0.2. Structure of results and discussion. In the first two sections, we provide a comprehensive overview of how fNIRS-neurofeedback training is implemented, describing and discussing important features of neurofeedback-training protocols (1) and of the real-time signalprocessing methods applied (2). In the third section, we critically evaluate the quality of published studies including experimental design and reporting quality as well as statistical power/sensitivity as an indicator of reliability of the reported findings (3). In the fourth section, we assess and discuss the effectiveness of fNIRS-neurofeedback to regulate and induce pre-post changes in brain activity (4). Finally, we assess and discuss its effectiveness in changing behavioral outcomes and we review the clinical potential of fNIRS-neurofeedback (5). The fNIRS illustration was created by Laura Bell.

\section{Training protocols}

When designing a neurofeedback study and creating a new training protocol, there are a number of aspects to be considered. Some of these include: (1) population(s) to be studied (e.g., healthy or patients or both), (2) neural target for the neurofeedback training (e.g., a particular [set of] brain region(s) or a measure of connectivity), (3) control-group approach (see Section 3.1), (4) duration of training, and (5) the neurofeedback procedure, including training conditions (e.g., regulation, rest, transfer), the kind of feedback presentation and task instructions. In this section of the review, we provide an overview of how previous fNIRS-neurofeedback studies established these protocol aspects. We also discuss implications and possible extensions for future studies. Table 1 shows details of the training protocols used in the studies included in our review. 


\subsection{Target populations}

Figure 1A shows the different target populations investigated in the studies. Mostly healthy participants were investigated $(\mathrm{N}=337)$, but also patients after stroke $(\mathrm{N}=20)$, with social anxiety disorder $(\mathrm{N}=12)$, autism spectrum disorder $(\mathrm{N}=6)$, attention-deficit/hyperactivity disorder $(\mathrm{N}=27$ children and $\mathrm{N}=19$ adults), and adults with high impulsivity $(\mathrm{N}=20)$.

\subsection{Target regions}

Figure 1B shows the distribution of the neurofeedback target regions of the included studies. The bulk of the studies trained participants to regulate parts of the prefrontal cortex (PFC), i.e., dorsolateral prefrontal cortex (dlPFC), inferior frontal gyrus (IFG), frontal pole or orbitofrontal cortex (OFC). Some studies broadly targeted the PFC using eight to fourteen target channels (Barth et al., 2016; Hudak et al., 2017, 2018; Kimmig et al., 2018) and others targeted specific subregions of the PFC using only one target channel (Li et al., 2019). Another large proportion of the studies trained participants to regulate activation within sensorimotor regions and enhance motor imageryrelated brain activation (e.g., Fujimoto et al., 2017). One study reinforced up-regulation of temporal and frontal face-processing regions, as individually defined by a functional localizer (Liu et al., 2016) and another aimed to train a broad affective network of frontal and occipital brain regions using a multivariate classifier approach (Trambaiolli et al., 2018). The signal-to-noise (SNR) ratio likely differs between target regions and depends on scalp-brain or source-detector distance. The SNR is dependent on individual physical features such as individual brain anatomy, head size, skull thickness, and hair properties (e.g., thickness, density, length and color; Orihuela-Espina et al., (2010)). On average, the scalp-brain distance is higher in parietal regions and lower in frontal and temporal regions (Cui et al., 2011). These factors should be taken into account when selecting target regions/channels for a neurofeedback study (see also 2.2). It should be noted that to date no study has used fNIRS-neurofeedback training to target connectivity between specific brain regions.

\section{A. Target population}

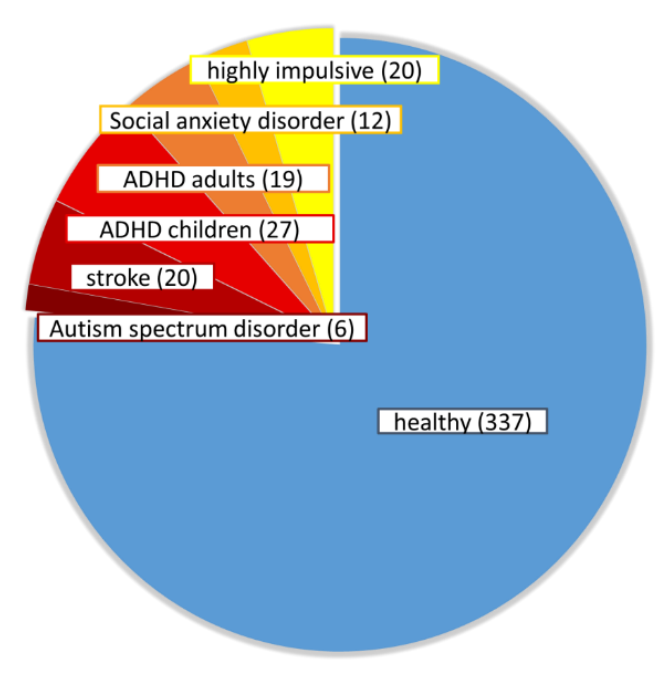

B. Target regions

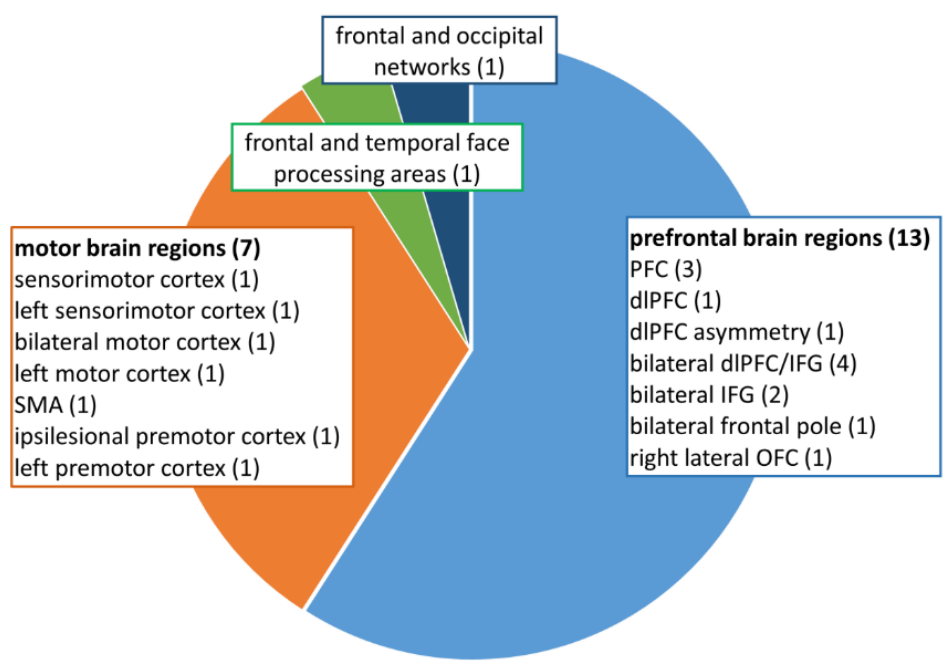

Figure 1. (A) Number of participants from different target populations and (B) Number of studies targeting a certain brain region. $\mathrm{ADHD}$, attention-deficit/hyperactivity disorder; dlPFC,dorsolateral prefrontal cortex; IFG, inferior frontal gyrus; OFC, orbitofrontal cortex; PFC, prefrontal cortex; SMA, supplementary motor area. 
Table 1. Neurofeedback training protocol details.

\begin{tabular}{|c|c|c|c|c|c|c|}
\hline \multirow[t]{2}{*}{ Study } & \multirow{2}{*}{$\begin{array}{l}\text { Data for Section } \\
1.2 \text { and Fig. } 1.1 \mathrm{~B} \\
\text { Target }\end{array}$} & \multirow{2}{*}{\begin{tabular}{|l|} 
Data for Section 1.3 \\
Training
\end{tabular}} & \multicolumn{4}{|l|}{ Data for Section 1.4} \\
\hline & & & $\begin{array}{l}\text { Neurofeedback run periods and } \\
\text { timing }\end{array}$ & Feedback & Instructed strategies & $\begin{array}{l}\text { Regulation } \\
\text { (total time) }\end{array}$ \\
\hline $\begin{array}{l}\text { Aranyi et al. } \\
\text { (2016) }\end{array}$ & $\begin{array}{l}\text { bilateral dIPFC } \\
\text { asymmetry }\end{array}$ & $\begin{array}{l}1 \text { session / } 1 \text { practice run, } 8 \text { real runs / } 1 \text { trial, } 1 \\
\text { day of training }\end{array}$ & $\begin{array}{l}15 \mathrm{~s} \text { rest } / 3 \mathrm{~s} \text { instruction } / 40 \mathrm{~s} \\
\text { mental counting baseline / } 10 \mathrm{~s} \text { rest / } \\
3 \mathrm{sinstruction} / 40 \mathrm{~s} \text { regulation } / 7 \mathrm{~s} \\
\text { rest }\end{array}$ & $\begin{array}{l}\text { Immediate visual feedback, engagement } \\
\text { (e.g., gaze, smile) of virtual agent }\end{array}$ & $\begin{array}{l}\text { express positive } \\
\text { feelings towards the } \\
\text { agent in order to } \\
\text { capture its interest }\end{array}$ & $6 \mathrm{~min}$ \\
\hline $\begin{array}{l}\text { Barth et al. } \\
\text { (2016) }\end{array}$ & PFC & $\begin{array}{l}8 \text { sessions / } 1 \text { run / } 12 \text { trials / } 30 \text { s / } 8 \text { days over } \\
2 \text { weeks }\end{array}$ & $\begin{array}{l}30 \text { s relaxation (deactivation) / } 30 \mathrm{~s} \\
\text { regulation (activation) }\end{array}$ & $\begin{array}{l}\text { Immediate visual feedback of all channels } \\
\text { (color-coded) }\end{array}$ & not reported & $48 \mathrm{~min}$ \\
\hline $\begin{array}{l}\text { Fujimoto et al. } \\
\text { (2017) }\end{array}$ & SMA & $\begin{array}{l}1 \text { session real and } 1 \text { session sham NF/ } 16 \text { trials / } \\
5 \mathrm{~s} / 2 \text { days }\end{array}$ & $8-15 \mathrm{~s}$ rest/ $5 \mathrm{~s}$ regulation & $\begin{array}{l}\text { Immediate visual feedback, height and color } \\
\text { of vertical bar }\end{array}$ & no strategies instructed & $80 \mathrm{~s}$ \\
\hline $\begin{array}{l}\text { Hosseini et al. } \\
\text { (2016) }\end{array}$ & dIPFC & $\begin{array}{l}4 \text { sessions NF during verbal working memory } \\
\text { task / } 1 \text { run / } 80 \text { trials / } 4 \text { days over } 2 \text { weeks }\end{array}$ & $\begin{array}{l}8-10 s \text { working memory trial } \\
\text { (encoding and retention) / } 2 \mathrm{~s} \text { test / } \\
6-8 \mathrm{~s} \text { rest and feedback presentation }\end{array}$ & $\begin{array}{l}\text { Delayed feedback, line plot displaying } \\
\text { changes during previous ten trials }\end{array}$ & $\begin{array}{l}\text { meta-cognitive } \\
\text { strategies. }\end{array}$ & $48 \mathrm{~min}$ \\
\hline $\begin{array}{l}\text { Hudak et al. } \\
\text { (2017) }\end{array}$ & $\begin{array}{l}\text { bilateral } \\
\text { dIPFC/IFG }\end{array}$ & $\begin{array}{l}8 \text { sessions / } 2 \text { runs } N F / 12 \text { trials / } 1 \text { run transfer } \\
\text { / } 8 \text { trials / session 1-4: 50/50\%, session 5-8: } \\
80 / 20 \% \text { activation/deactivation trials / } 8 \text { days } \\
\text { over } 2 \text { weeks }\end{array}$ & $\begin{array}{l}20 \mathrm{~s} \text { rest }(5 \mathrm{~s} \text { baseline }) / 30 \mathrm{~s} \\
\text { regulation } / 2 \mathrm{~s} \text { reward }\end{array}$ & $\begin{array}{l}\text { Immediate visual feedback, virtual } \\
\text { classroom scenario: brightness of the } \\
\text { lighting in the classroom, reinforcement } \\
\text { after each trial }\end{array}$ & no strategies instructed & $\begin{array}{l}96 \min +32 \\
\min \text { transfer }\end{array}$ \\
\hline $\begin{array}{l}\text { Hudak et al. } \\
\text { (2018) }\end{array}$ & $\begin{array}{l}\text { bilateral } \\
\text { dIPFC/IFG }\end{array}$ & $\begin{array}{l}30 \text { sessions / } 2 \text { runs NF / } 12 \text { trials / } 1 \text { run } \\
\text { transfer / } 8 \text { trials / } 50 / 50 \% \\
\text { activation/deactivation trials / } 30 \text { days over } 12 \text { - } \\
49 \text { weeks, } 3 \text { weeks intermission after session } 15\end{array}$ & $\begin{array}{l}30 \text { rest }(5 \mathrm{~s} \text { baseline }) / 30 \mathrm{~s} \text { regulation } \\
/ 2 \mathrm{~s} \text { reward }\end{array}$ & $\begin{array}{l}\text { Immediate visual feedback was presented } \\
\text { via commercial EEG-NF system (moving } \\
\text { objects and } 2 \text { s reinforcement) }\end{array}$ & not reported & $\begin{array}{l}360 \mathrm{~min}+ \\
120 \mathrm{~min} \\
\text { transfer }\end{array}$ \\
\hline $\begin{array}{l}\text { Kanoh et al. } \\
\text { (2011) }\end{array}$ & $\begin{array}{l}\text { left sensorimotor } \\
\text { cortex }\end{array}$ & $\begin{array}{l}5 \text { sessions } / 6 \text { runs } / 5 \text { trials } / 20 \mathrm{~s} / 5 \text { days of } \\
\text { training }\end{array}$ & $40-43$ s rest / 20 s up-regulation & $\begin{array}{l}\text { Immediate visual feedback (length of white } \\
\text { bar) }\end{array}$ & $\begin{array}{l}\text { motor imagery of right } \\
\text { hand }\end{array}$ & $50 \mathrm{~min}$ \\
\hline $\begin{array}{l}\text { Kimmig et al. } \\
\text { (2018) }\end{array}$ & $\begin{array}{l}\text { bilateral } \\
\text { dIPFC/IFG }\end{array}$ & $\begin{array}{l}15 \text { sessions / } 2 \text { runs } N F / 12 \text { trials / } 1 \text { run } \\
\text { transfer / } 8 \text { trials / } 75 / 25 \% \text { up-/downregulation } \\
\text { / } 15 \text { days over } 5-9 \text { weeks, from } 7^{\text {th }} \text { session: } \\
\text { distractor background pictures with fear-related } \\
\text { contents }\end{array}$ & $\begin{array}{l}30 \mathrm{~s} \text { rest }(5 \mathrm{~s} \text { baseline }) / 30 \mathrm{~s} \\
\text { regulation / } 2 \mathrm{~s} \text { reward }\end{array}$ & $\begin{array}{l}\text { Immediate visual feedback, moving dot, and } \\
\text { reinforcement "Well done!", anxiety-related } \\
\text { or neutral background pictures from } 7^{\text {th }} \\
\text { session onwards }\end{array}$ & not reported & $\begin{array}{l}180 \mathrm{~min}+ \\
60 \text { min } \\
\text { transfer }\end{array}$ \\
\hline $\begin{array}{l}\text { Kinoshita et al. } \\
\text { (2016) }\end{array}$ & $\begin{array}{l}\text { bilateral frontal } \\
\text { pole cortex }\end{array}$ & $\begin{array}{l}1 \text { session / } 6 \text { runs ( } 2 \text { runs real / } 2 \text { runs sham NF / } \\
2 \text { runs transfer) } 18 \text { trials / } 1 \text { day of training }\end{array}$ & $16 \mathrm{~s}$ rest / $10 \mathrm{~s}$ up-regulation & Immediate visual feedback (blue bar) & $\begin{array}{l}\text { memory, executive } \\
\text { functions, and verbal } \\
\text { fluency strategies } \\
\text { suggested }\end{array}$ & $\begin{array}{l}6 \text { min real + } \\
6 \text { min } \\
\text { transfer }\end{array}$ \\
\hline $\begin{array}{l}\text { Kober et al. } \\
\text { (2014) }\end{array}$ & $\begin{array}{l}\text { motor cortex } \\
\text { asymmetry }\end{array}$ & 8 sessions / 2 runs / 40 trials / 8 different days & $7-11$ s rest / $6-8 \mathrm{~s}$ regulation & $\begin{array}{l}\text { Immediate visual feedback, moving dot + } \\
\text { numerical score continuously updated }\end{array}$ & $\begin{array}{l}\text { kinesthetic motor } \\
\text { imagery }\end{array}$ & $\sim 75 \mathrm{~min}$ \\
\hline $\begin{array}{l}\text { Kober et al. } \\
(2015)\end{array}$ & bilateral IFG & 7 sessions / 1 run / 25 trials / 7 different days & $27-33$ s rest / $17-23$ s up-regulation & $\begin{array}{l}\text { Immediate visual feedback, moving dot }+ \\
\text { numerical score continuously updated }\end{array}$ & motor imagery & $\sim 58 \mathrm{~min}$ \\
\hline $\begin{array}{l}\text { Kober et al. } \\
\text { (2018) }\end{array}$ & bilateral IFG & $\begin{array}{l}1 \text { session / } 1 \text { run / } 20 \text { trials / } 1 \text { day (also NF } \\
\text { during rest, but instructed to relax and bring } \\
\text { signal back to baseline) }\end{array}$ & $30 \mathrm{~s}$ rest / $17-23 \mathrm{~s}$ regulation & $\begin{array}{l}\text { Immediate visual feedback (color-coded on } \\
\text { a schematic head model) }\end{array}$ & $\begin{array}{l}\text { kinesthetic motor } \\
\text { imagery }\end{array}$ & $\sim 7 \mathrm{~min}$ \\
\hline
\end{tabular}


fNIRS-Neurofeedback - a systematic review

Table 1 (continued)

\begin{tabular}{|c|c|c|c|c|c|c|}
\hline \multirow[t]{2}{*}{ Study } & \multirow{2}{*}{$\begin{array}{l}\text { Data for Section } \\
1.2 \text { and Fig. 1.1 B } \\
\text { Target }\end{array}$} & \multirow{2}{*}{$\begin{array}{l}\text { Data for Section } 1.3 \\
\text { Training }\end{array}$} & \multicolumn{4}{|l|}{ Data for Section 1.4} \\
\hline & & & $\begin{array}{l}\text { Neurofeedback run periods and } \\
\text { timing }\end{array}$ & Feedback & Instructed strategies & $\begin{array}{l}\text { Regulation } \\
\text { (total time) }\end{array}$ \\
\hline $\begin{array}{l}\text { Lapborisuth et } \\
\text { al. (2017) }\end{array}$ & left motor cortex & $\begin{array}{l}1 \text { session / } 8 \text { runs (motor imagery and } \\
\text { execution) / } 4 \text { runs with NF / } 4 \text { runs without / } 6 \\
\text { trials }\end{array}$ & $15 \mathrm{~s}$ rest $/ 15 \mathrm{~s}$ up-regulation & $\begin{array}{l}\text { Immediate visual feedback of all channels } \\
\text { on a color-coded topographic image }\end{array}$ & $\begin{array}{l}\text { motor imagery / motor } \\
\text { execution }\end{array}$ & $6 \mathrm{~min}$ \\
\hline Lee et al. (2015) & $\begin{array}{l}\text { sensory motor } \\
\text { cortex }\end{array}$ & $\begin{array}{l}1 \text { session, } 2 \text { runs, } 1 \text { run treadmill walking } \\
\text { without } N F, 1 \text { run with } N F\end{array}$ & $15 \mathrm{~s}$ rest $/ 10 \mathrm{~s}$ up-regulation & Immediate visual feedback (red bar) & not reported & $100 \mathrm{~s}$ \\
\hline Li et al. (2019) & right lateral OFC & $\begin{array}{l}1 \text { session, } 1 \text { run } 6-10 \text { trials to learn strategy, } 4 \\
\text { real training runs / } 4 \text { trials / one day / 10-30 min } \\
\text { break in between }\end{array}$ & $25 \mathrm{~s}$ rest $/ 25 \mathrm{~s}$ up-regulation & $\begin{array}{l}\text { Immediate visual feedback, animation: "Lift } \\
\text { a stone in front of a beach landscape" }\end{array}$ & $\begin{array}{l}\text { no specific strategies } \\
\text { instructed }\end{array}$ & $\sim 9-11 \mathrm{~min}$ \\
\hline Liu et al. (2016) & $\begin{array}{l}\text { frontal and } \\
\text { temporal face } \\
\text { processing regions }\end{array}$ & $\begin{array}{l}5 \text { sessions / } 2 \text { runs / } 1 \text { run: functional localizer / } \\
4 \text { trials morphing faces / } 1 \text { run training / } 10 \\
\text { trials, } 5 \text { days over } 5 \text { weeks }\end{array}$ & $\begin{array}{l}30 \mathrm{~s} \text { rest / house-matching } 20 \mathrm{~s} / \\
\text { face-matching (up-regulation) } 28 \mathrm{~s} / \\
2 \mathrm{~s} \text { feedback/reward display }\end{array}$ & $\begin{array}{l}\text { Delayed feedback, points displayed after } \\
\text { each trial, points were later converted to } \\
\text { cash }\end{array}$ & $\begin{array}{l}\text { no specific strategies } \\
\text { instructed }\end{array}$ & $\sim 23 \min$ \\
\hline $\begin{array}{l}\text { Marx et al. } \\
\text { (2015) }\end{array}$ & $\begin{array}{l}\text { bilateral } \\
\text { dIPFC/IFG }\end{array}$ & $\begin{array}{l}12 \text { sessions / } 2 \text { runs } N F / 12 \text { trials / } 1 \text { run transfer } \\
\text { / } 8 \text { trials, } 50 / 50 \% \text { activation/deactivation trials, } \\
12 \text { days within } 4-6 \text { weeks }\end{array}$ & $\begin{array}{l}25 \mathrm{~s} \text { rest }(5 \mathrm{~s} \text { baseline) / } 30 \mathrm{~s} \\
\text { regulation } / 2 \mathrm{~s} \text { reward }\end{array}$ & $\begin{array}{l}\text { Immediate visual feedback was presented } \\
\text { via commercial EEG-NF system (moving } \\
\text { objects and } 2 \mathrm{~s} \text { reinforcement) }\end{array}$ & not reported & $\begin{array}{l}144 \min + \\
48 \text { min } \\
\text { transfer }\end{array}$ \\
\hline $\begin{array}{l}\text { Mihara et al. } \\
\text { (2012) }\end{array}$ & $\begin{array}{l}\text { left premotor } \\
\text { cortex }\end{array}$ & $\begin{array}{l}1 \text { session real / } 1 \text { session sham NF / } 15 \text { trials / } 1 \\
\text { day }\end{array}$ & $8-15 \mathrm{~s}$ rest $/ 5 \mathrm{~s}$ regulation & $\begin{array}{l}\text { Immediate visual feedback, height and color } \\
\text { of vertical bar }\end{array}$ & $\begin{array}{l}\text { kinesthetic motor } \\
\text { imagery }\end{array}$ & $75 \mathrm{sec}$ \\
\hline $\begin{array}{l}\text { Mihara et al. } \\
\text { (2013) }\end{array}$ & $\begin{array}{l}\text { ipsilesional } \\
\text { premotor cortex }\end{array}$ & $\begin{array}{l}6 \text { sessions / } 32 \text { trials / } 6 \text { days over } 2 \text { weeks / } \\
10 \text { min motor imagery training without NF } \\
\text { before each session }\end{array}$ & $8-15 \mathrm{~s}$ rest $/ 5 \mathrm{~s}$ regulation & $\begin{array}{l}\text { Immediate visual feedback, height and color } \\
\text { of vertical bar }\end{array}$ & $\begin{array}{l}\text { kinesthetic motor } \\
\text { imagery }\end{array}$ & $16 \mathrm{~min}$ \\
\hline Narita (2015) & left PFC & $\begin{array}{l}2 \text { sessions / } 7 \text { runs / } 6 \text { trials / } 2 \text { days over } 1 \\
\text { week? (but not clearly reported) }\end{array}$ & $15 \mathrm{~s}$ rest $/ 30 \mathrm{~s}$ regulation & Immediate visual feedback, color of monitor & not reported & $\sim 42 \min$ \\
\hline $\begin{array}{l}\text { Trambaiolli et } \\
\text { al. (2018) }\end{array}$ & $\begin{array}{l}\text { frontal and } \\
\text { occipital networks }\end{array}$ & $\begin{array}{l}1 \text { session / } 2 \text { runs classifier training / } 2 \text { runs NF / } \\
11 \text { trials ( } 5 \text { trials real, } 3 \text { trials fixed, } 3 \text { trials } \\
\text { random feedback (neutral and positive affect } \\
\text { conditions) }\end{array}$ & $\begin{array}{l}5 \mathrm{~s} \text { fixation cross } / 2 \mathrm{~s} \text { instruction / } 30 \\
\mathrm{~s} \text { positive or neutral affect condition } \\
\text { / self-paced self-evaluation }\end{array}$ & $\begin{array}{l}\text { Immediate visual feedback, amorphous } \\
\text { figure }\end{array}$ & $\begin{array}{l}\text { imagine positive } \\
\text { personal experiences }\end{array}$ & $5 \mathrm{~min}$ \\
\hline $\begin{array}{l}\text { Weyand et al. } \\
\text { (2015) }\end{array}$ & bilateral PFC & $\begin{array}{l}16 \text { sessions / } 3 \text { runs / } 20-22 \text { trials (up- and } \\
\text { down-regulation) / } 15 \text { sessions within } 3 \text { weeks, } \\
\text { last session } 10 \text { days later / session 1-5: select } \\
\text { strategy / session 6-10: practice strategies / } \\
\text { session 11-15: stop strategies, use desire to } \\
\text { regulate / session 16: follow-up }\end{array}$ & 20 s rest $/ 17$ s regulation & $\begin{array}{l}\text { Immediate visual feedback, color-coded } \\
\text { topographic image, ball that rises and falls, } \\
\text { and game feedback }\end{array}$ & $\begin{array}{l}\text { yes, specific strategies } \\
\text { instructed }\end{array}$ & $277 \mathrm{~min}$ \\
\hline
\end{tabular}




\subsection{Training duration}

The length of training varied broadly between studies and ranged from short one-session designs (nine studies) up to 30 sessions (Hudak et al., 2018), resulting in a total duration of regulation training that varied between $75 \mathrm{~s}$ and up to 8h.In particular, clinical treatment studies used a higher number of training sessions (Hudak et al., 2018; Kimmig et al., 2018; Marx et al., 2015). The majority of studies applied five sessions or less. While the optimal number of sessions for acquiring self-regulation of hemodynamic brain responses via fNIRS needs to be determined, successful regulation after even a single session has been reported in sham-controlled studies (Fujimoto et al., 2017; Li et al., 2019). Similar to fMRI-neurofeedback, fNIRS-neurofeedback targets spatially specific brain hemodynamics and might therefore offer a faster pace of learning compared to EEGneurofeedback with most studies involving 20-40 training sessions (see also Marx et al., 2015; Thibault et al., 2018). However, the data of studies by (Marx et al., 2015; Mayer et al., 2015) that directly compared fNIRS- with EEG-neurofeedback still need to be published to shed further light on different learning mechanisms.

\subsection{Neurofeedback procedure}

A neurofeedback procedure consists of at least three different aspects: (1) within-run task periods and their timing, (2) feedback presentation (i.e., sensory modality employed, timing, and complexity of feedback information, and (3) instructions provided to participants.

\subsubsection{Neurofeedback run periods and their timing}

A neurofeedback run procedure consists of at least two different kinds of periods, i.e., a regulation period during which participants receive neurofeedback and try to change brain activity, and a resting period during which no feedback is provided that can also serve as a baseline-control condition. Most studies instructed participants to rest during the control condition, except for one study that instructed participants to engage in mental counting in an attempt to control for potentially confounding mental processes (Aranyi et al., 2016). Some protocols also included additional reward periods (e.g., smiling faces or points) presented after each regulation trial (Hudak et al., 2017, 2018; Kimmig et al., 2018; Liu et al., 2016), which is a form of additional delayed feedback. Two studies applied a combination of neurofeedback with (socio-)cognitive training and presented delayed feedback on activity during a cognitive task after a block (Hosseini et al., 2016; Liu et al., 2016). To ensure or assess the transfer of self-regulation skills beyond the neurofeedback sessions, a few studies employed transfer (nofeedback) periods (Hudak et al., 2017, 2018; Kimmig et al., 2018; Marx et al., 2015), where participants received the same instructions as in the neurofeedback task, but without receiving any feedback on their brain activity. Unfortunately, these studies did not report regulation success specifically for transfer periods. While most protocols either trained up- or downregulation, some also trained regulation in both directions, where activation and deactivation periods were randomly presented. The lengths of the regulation and baseline conditions varied broadly across studies from 5$40 \mathrm{~s}$ to 6-43 s, with most studies varying between $20-30 \mathrm{~s}$.

Transfer indicates that a skill is transferred to different situations or tasks. Depending on the contextual factors of the transfer situation, we can distinguish between near and far transfer, with the latter being more important for the success of training (see Barnett and Ceci, 2002). However, some studies (Hudak et al., 2017, 2018; Kimmig et al., 2018; Marx et al., 2015) used the term transfer to describe task periods but presented a reward after each transfer trial. Hence, they switched from immediate to delayed neurofeedback training and are unable to ensure or demonstrate that 
participants are able to regulate brain activity without receiving feedback, i.e. to transfer the skill of brain regulation to a new situation and possibly beyond neurofeedback training (far transfer). When combining up- and downregulation within a session, care should be taken to balance the randomization of up- and down-regulation periods in order to keep transition probabilities between periods equal. Otherwise, participants may anticipate and prepare for the following condition, i.e., regulate in the opposing direction during the baseline condition as demonstrated by Hudak et al. (2018). Similarly, anticipatory effects might be prevented by introducing variable onsets of the regulation conditions, as applied by some of the studies. This would render randomization of periods unnecessary and enable to present several up- and downregulation periods in a blocked fashion, which reduces cognitive demands and may facilitate shaping of individual strategies during the training. However, combining up- and downregulation in a single session might involve the risk of carry-over effects between periods, which may impede regulation performance. However, this speculation needs to be corroborated by a comparative study. The optimal trial structure for neurofeedback procedures needs further investigation. Since comparative studies including fMRIand EEG-neurofeedback research are lacking, we mostly rely on theoretical considerations when designing neurofeedback tasks. Considering the time course of the hemodynamic response (Ogawa et al., 1992), which is delayed and peaks after 4-6 s, a reasonable duration of the regulation and baseline period is 20-30 s. Further research is needed to discover whether shorter or longer periods of up to $40 \mathrm{~s}$ (Aranyi et al., 2016) and above are beneficial. While a short duration of $5 \mathrm{~s}$ can be considered too short for a hemodynamic response to develop properly, these studies continue to show the feedback signal during a subsequent rest period (Fujimoto et al., 2017; Mihara et al., 2012, 2013). Moreover, we can assume that if feedback is presented immediately (a slowly developing hemodynamic signal), longer durations are required compared to delayed feedback, which is presented considering the activation of a whole previous trial.

\subsubsection{Feedback presentation}

The presentation of feedback information may differ with regard to (1) sensory modality (visual, auditory, tactile), (2) timing (immediate vs. delayed), (3) complexity (simple vs. complex virtual environment), and (4) rewarding content (smiling faces or monetary reward). Most of the studies used a simple type of immediate visual feedback in the form of a bar (e.g., Fujimoto et al., 2017) or more complex animations of, for example, rising objects (e.g., Li et al., 2019; Marx et al., 2015), or smiling virtual agents (Aranyi et al., 2016). Others used color-coded topographic maps (Barth et al., 2016; Kober et al., 2018; Lapborisuth et al., 2017), displaying the signal change of all channels while instructing participants to focus on a certain region within the channel arrangement. Kimmig et al., (2018) started with a simple form of visual feedback (moving ball) and introduced neutral and anxiety-related pictures in the middle of the training period to provide a relevant context for patients with social anxiety disorder. Some studies presented delayed feedback in addition to immediate feedback in the form of a reward (e.g., smileys or points) after each trial (Hudak et al., 2017; Kimmig et al., 2018), or only delayed feedback in order to reduce distraction during the task/regulation period (Hosseini et al., 2016; Liu et al., 2016).

Whether immediate or delayed feedback is superior or whether they are equally effective is a matter of ongoing debate and there is only limited and as yet unclear evidence from a few comparative fMRI-neurofeedback studies (see Paret et al., 2019). In case of the noisier fNIRSneurofeedback, feeding back mean or median activity at the end of each block may avoid confusing participants due to noisy fluctuations of the feedback signal and may also be beneficial with regard to timing (see above). The form of social neurofeedback used by Aranyi et al., (2016) may be experienced as more rewarding and may improve motivation, which is confirmed by preliminary 
evidence from an fMRI-neurofeedback study demonstrating that social neurofeedback outperforms simple visual feedback and leads to stronger activation of reward-related brain regions (Mathiak et al., 2015). In an effort to increase motivation and facilitate transfer to daily life or critical situations, neurofeedback has been embedded in a 3D virtual-reality environment. While the feasibility of this approach has been demonstrated in a subclinical adult population (Hudak et al., 2017), an ongoing clinical trial in children with attention-deficit/hyperactivity disorder (ADHD) is investigating whether virtual-reality-based neurofeedback is superior to simpler forms of feedback (Blume et al., 2017). Some researchers are opposed to complex feedback, arguing that neurofeedback should lead to knowledge of results and that gaming environments which are too complex may distract participants (Hinterberger et al., 2004; Sherlin et al., 2011). However, comparative studies provide evidence that the more engaging complex feedback embedded in a virtual environment may facilitate learning and is better received by users (Cohen et al., 2016; Gruzelier et al., 2010).

\subsubsection{Instructions}

In a neurofeedback study, participants are provided with general task instructions about the experiment and in most cases also instructions on how to regulate a certain brain region. Instructions can be very specific (e.g., use motor imagery to regulate motor brain regions) or rather loose (e.g., use mental strategies to change the feedback signal).

Twelve studies instructed participants to upregulate, one to downregulate, and six to up- and downregulate a certain target region. Another study reinforced asymmetry, i.e., a difference between the activity of a target region and its homologue on the other hemisphere, and two used a multivariate analysis approach to calculate the feedback signal. While some studies provided explicit instructions about types of mental strategies to regulate brain activity, such as kinesthetic motor imagery (Kober et al., 2014) or affective strategies (Trambaiolli et al., 2018), others merely encouraged participants to learn self-regulation using a trial-and-error approach (e.g., Fujimoto et al., 2017; Hudak et al., 2017).

Even if no strategies are provided, study design and general instructions about the experiment may prime the use of certain strategies (Kohl et al., 2019). For example, in the study by Fujimoto et al., (2017), when participants were informed about the target region (supplementary motor area) they might have been tempted to use motor-imagery strategies. Therefore, care needs to be taken when giving task instructions and also when informing participants about the general purpose of the experiment. It is thus important that participants' strategies are documented thoroughly, ideally after each neurofeedback trial or run (see also 3.2), although results need to be interpreted carefully, since retrospective self-reports may lack realiability (see Veenman, 2011), particularly in children (Stone and Lemanek, 1990). Whether explicit instructions are beneficial for neurofeedback learning or not remains an open question and may depend on the particular training protocol (see Paret et al., 2019). For instance, it has been suggested that explicit strategies are not necessary, and some work even indicated that they may be detrimental or at least not helpful in some cases (Birbaumer et al., 2013; Sepulveda et al., 2016; Shibata et al., 2019). This is also supported by a recent fNIRS-neurofeedback study (Weyand et al., 2015) where participants used two personalized mental strategies over ten sessions and were then instructed to stop using their strategies and instead to use only their desire to regulate brain activity. Interestingly, regulation performance remained the same after weaning off specific mental strategies and the majority of participants reported training to be less demanding and more intuitive. Nevertheless, strategy instruction may initially facilitate learning (Scharnowski et al., 2015) and may be helpful if successful strategies to regulate a certain brain region are clearly known, e.g., using motor imagery to regulate motor regions. Hence, the decision about instructions may depend on the targeted brain region, duration, and purpose of the neurofeedback-training experiment. 
fNIRS-Neurofeedback - a systematic review

\subsection{Conclusion - Training protocols}

FNIRS-neurofeedback has been applied in a variety of populations, including different patient populations, children, adolescents, and older adults. However, previous studies are heterogeneous in terms of (i) selected neurofeedback targets (mostly comprising prefrontal and sensory motor brain regions), (ii) duration of training, and (iii) design of the neurofeedback procedure, including timing, feedback display, and instructions. Generally, as fNIRS measures the same hemodynamic brain signal as fMRI, a large part of the fMRI-neurofeedback training procedures can be (and have already been) transferred to fNIRS-neurofeedback. Note, however, that also for neurofeedback in general there are a lot of open issues with regard to training protocol methods (see Paret et al., 2019). Further systematic research and discussion will help to achieve consensus and make neurofeedback training protocols more efficient.

\section{Online signal-processing methods and hardware}

In this section, we give an overview of the different methods used for online signal-processing, including devices, selection of brain regions (fNIRS channels) of interest, online feature extraction (chromophores used), and preprocessing and artifact control (see also Table 2). Providing valid feedback of neural activity to the participant is a crucial component of successful neurofeedback applications. Therefore, methods have to be carefully selected to capture the neural activity of a target region and minimize strong extracranial artifacts, as are frequently present in the fNIRS signal (Caldwell et al., 2016).

\subsection{Devices}

Nine different devices were used in the studies: ETG-4000, OMM-3000, NIRSport, NIRScout, fNIR400, LABNIRS, FOIRE-3000, PocketNIRS, and Imagent. Some devices were used more frequently than others. However, this does not imply qualitative superiority, and it is beyond the scope of this review to judge the functionality of the devices used. Notably, other commercially available devices are used in cognitive neuroscience and custom-developed mobile fNIRS instrumentations (also available open-source: www.opennirs.org) are used for BCI applications (von Lühmann et al., 2015), and may be used for neurofeedback applications. For an overview of commercially available fNIRS systems and their features refer to Pinti et al. (2018a) and Scholkmann et al. (2014). Major differences between the devices are the wavelength used and the amount of wavelength exploited by the systems. Five of the devices used two wavelengths (ETG-4000, NIRSport, NIRScout, Imagent, fNIR400) whereas the other four devices support three wavelengths (OMM-3000, FOIRE-3000, LABNIRS, PocketNIRS). It should be noted that each of the devices used different wavelengths in the range from $690 \mathrm{~nm}$ to $860 \mathrm{~nm}$ and had different precisions (e.g., dynamic range or sensitivity, based on the hardware and its quality). These differences can potentially constitute confounds in the context of reproducibility of neurofeedback results. The use of more wavelengths can improve signal quality (Arifler et al., 2015) and might therefore be beneficial for fNIRS-neurofeedback applications.

\subsection{Selection of target regions (channels of interest)}

In order to target specific brain regions reliably, the studies applied different methods to verify appropriate channel selection for extracting the neurofeedback information. In fNIRS-neurofeedback studies, a certain number of optodes are placed on the participants' heads (general optode setup), then some of the channels ('channels of interest') are selected to extract the neurofeedback information. All the studies used a priori knowledge about the assumed location of specific brain regions involved in the regulation task. Additionally, most studies used the EEG 10-20 system (Jasper, 1958) as a 
reference to place the optodes (20 out of 22 studies; Table 2) and the methods of Okamoto et al. (2004), Singh et al. (2005) or Tsuzuki et al. (2007) to register channels to the MNI space.

Alternatively (or in combination), the positions were either verified by an individual or reference structural MRI scan combined with a digitizer measurement (Fujimoto et al., 2017) or a functional localization procedure was performed. Eight studies used additional digitizer measurements to verify channel positions post hoc. Three studies used a functional localizer before the training session to select task-relevant channel(s) with individually good signal quality (Hosseini et al., 2016; Kober et al., 2014; Liu et al., 2016). In one study, the optodes were placed on the forehead of the subject without describing the use of other reference points (Aranyi et al., 2016). Three studies provided no further information about additional registration of the optodes. For the eight studies using digitizer measurements, the channels were positioned with respect to the neurofeedback target region based on MNI coordinates using virtual spatial registration or general head location in combination with a functional localizer (Liu et al., 2016). The input for the online feature was either a single channel or the average signal of channels covering the region of interest. The number of channels used for extracting the neurofeedback information ranged from 1 to 14 for studies using amplitude changes and 9 to 32 channels for studies using multivariate statistics as a feature for the feedback (see Table 2).

In sum, the selection of channels of interest relied mainly on the EEG 10-20 system in combination with MNI coordinates and a priori knowledge about specific brain regions that are involved in the regulation task, which is a suitable procedure for selecting target channels. A combination with functional localizers seems to be a more reliable solution (if applicable) for amplitude-based studies since it additionally takes subject-specific variance into account by selecting individualized channels. Additionally, using digitization of optode locations and alignment with (preferably individual) MRI scans allows even more details to be obtained on optimum placement of the optodes.

\subsection{Online preprocessing and artifact control}

The fNIRS signal comprises different sources of noise. Most problematic in the context of real-time analysis seems to be the physiological noise that overlaps partly with task frequency, such as lowfrequency oscillations of blood pressure (Mayer waves; Kamran et al. (2016)). The studies applied different online preprocessing methods to deconfound the feedback signal from these sources of noise. Five studies did not report the online preprocessing methods they used and are thus not further considered in this review with respect to their preprocessing (Barth et al., 2016; Kinoshita et al., 2016; Kober et al., 2018; Lee et al., 2015; Narita, 2015). The majority of the studies which reported their online pre-processing steps applied different kinds of high- and low-pass filters (10 of 17; Table 2). Some studies did not use a high-pass filter (e.g., Aranyi et al., 2016; Kimmig et al., 2018; Li et al., 2019). For low-pass filtering, mainly finite impulse response (FIR) filters were used, most commonly a moving-average filter (window ranging from 2-5 s). Four studies (Hudak et al. $(2017,2018$ ), Kimmig et al. (2018), Marx et al. (2015)) used a common average reference (CAR) for data preprocessing, i.e., subtracting the average of all channels from the feedback channel, three of these in combination with additional filtering. Four studies used one or multiple reference channels (Aranyi et al., 2016; Kober et al., 2014, 2015; Liu et al., 2016). However, considering the lack of relevant information in other studies, it may be the case that some of these studies used additional filters but did not report them. Most of the studies did not use any explicit artifact control on top of the filtering methods ( 7 of 17). The studies that used artifact control to some degree most frequently employed either CAR or reference channels (8 out of 11) and one study (Kober et al., 2015) also included electromyography (EMG) measures for post hoc artifact control. Only one of these eight studies used 
short-distance channels to control for artifacts (in combination with EMG measures, Fujimoto et al. (2017)). Two studies used either only EMG (Mihara et al., 2013) or the respiration rate (Kinoshita et al., 2016) as a reference for post hoc artifact control, which was implemented using, for example, visual inspection of the EMG signal or differences in the respiration rate. Aranyi et al. (2016) used a sliding-window motion artifact rejection (SMAR) procedure, which rejected motion-affected periods in the fNIRS signal. Other motion correction methods suitable for real-time applications, e.g., Cui et al. (2010), were not applied.

Surprisingly, only three out of the nine studies that targeted motor regions used EMG to control for subtle movements (Fujimoto et al., 2017; Kober et al., 2015; Mihara et al., 2013), which can confound the feedback signal, and details of the EMG analysis are rarely reported. Other studies did not control for this confound or only visually inspected movements of the participants (Mihara et al., 2012). It is important that future studies targeting motor regions control for this confound. To date, studies have only looked at motion artifacts post hoc. Future studies could establish methods to control for EMG signals online, e.g., stop presenting feedback signal when movements occur or include the EMG signal as a nuisance regressor when using a general linear model (GLM) approach to calculate the feedback signal. Some studies did not apply high-pass filtering. It could be argued that if the feedback signal is compared to a preceding baseline in a short time frame, low-frequency drifts can be neglected, which would render high-pass filtering unnecessary. However, this remains to be confirmed by future research.

In general, the field would benefit from implementing more sophisticated artifact-control methods to account for potential confounding signals (Caldwell et al., 2016; Pfeifer et al., 2018; Tachtsidis and Scholkmann, 2016). Short-distance channels in combination with GLM seem to be the most efficient tool to correct for extracerebral physiological signal components (Brigadoi and Cooper, 2015; Tachtsidis and Scholkmann, 2016; von Lühmann et al., 2020). As already stated, only Fujimoto et al. (2017) used this technique, which may be because most of the fNIRS systems are not equipped with the appropriate hardware (Klein and Kranczioch, 2019). If this is the case, a potential alternative is the global component removal approach as introduced by Zhang et. al. (2016). This technique seems to be promising to reduce global physiological signals from the fNIRS data and can be used for online artifact control as recently pointed out by (Klein and Kranczioch, 2019) specifically with respect to single-trial data. FNIRS-neurofeedback studies have not yet applied this method but have rather used CAR or other referencing to correct for evoked systemic cerebral and extracerebral components. However, referencing should be applied with care, as reference channels have to be independent of the target region and participants may modulate the feedback signal by regulating reference channels instead of feedback channels (Hudak et. al., 2018). Therefore, the global component-removal approach could be a viable alternative. However, if none of those methods is available during the experiment it should be checked post hoc that these global signals did not drive the neurofeedback signal change.

\subsection{Online feature - Chromophores used}

For the online feature, either the amplitude or a derivative of the $\mathrm{HbO}$ or $\mathrm{HbR}$ was used, or a classifier was trained to discriminate specific states. Most studies (20 of 22) used a type of amplitude change. In examining the studies that used the amplitude, we found three studies (Fujimoto et al. (2017), Mihara et al. (2012) and Mihara et al. (2013)) which used the maximum t-value of the selected channels. All other studies used the HbO or HbR amplitude as a direct source for the feedback, scaled to a certain level, and referenced to a specific period before the feedback. For the studies using a classifier, a linear discriminant analysis (LDA) classifier was trained to discriminate two classes (e.g., neutral or positive affect, Trambaiolli et al. (2018), Weyand et al. (2015)). 
Even though there are multiple options for the source of neurofeedback information using fNIRS, most of the studies used HbO. Only two of the 22 studies used both chromophores and only two used HbR and $\mathrm{HbO}$ for different study groups (Kober et al., 2015, 2018). It is important to note that two of the four studies that used both chromophores employed a classifier approach (Trambaiolli et al., 2018), and one also included tHb (Weyand et al., 2015). All other studies used the direct amplitude of $\mathrm{HbO} / \mathrm{HbR}$ or a derivative of it. Kober et al. (2018) showed that people can regulate both chromophores with fNIRS-neurofeedback, but depending on the regulation strategy (in this case motor imagery to alter brain activation) regulation ability may be restricted to the natural course of the $\mathrm{HbO}$ and $\mathrm{HbR}$ signal changes related to this strategy.

The best-suited chromophore for neurofeedback (and other BCI) applications is still a matter of debate and has not been intensively investigated. Most studies use HbO since it displays larger amplitudes than HbR (Sato et al., 2016; Stangl et al., 2013). On the other hand, (Kirilina et al., 2012) reported HbR to be less sensitive to artifacts. However, a recent study (Klein and Kranczioch, 2019) demonstrated that $\mathrm{HbR}$ is also affected by a global signal component. The contrast-to-noise ratio seems to be comparable for $\mathrm{HbO}$ and $\mathrm{HbR}$ across different tasks (Cui et al., 2011), but according to Naseer and Hong (2015) HbO signals were more discriminative for BCI applications than those of HbR signals.

In sum, although less frequently used, current evidence does not imply that $\mathrm{HbR}$ or $\mathrm{tHb}$ are less suitable for fNIRS-neurofeedback. While all three options ( $\mathrm{HbO}, \mathrm{HbR}$ and $\mathrm{tHb}$ ) seem to be suitable, future research is still needed to ascertain whether one option outperforms the others in the context of neurofeedback applications.

\subsection{Calculation of feedback information}

The calculation of the feedback signal greatly depends on the type of display used for the presentation (see Section 1.4.2). Generally, the amplitude of the HbO signal during the feedback/task block was used and either compared to a preceding baseline (e.g., Hudak et al., 2017, 2018), the fNIRS-system baseline (Barth et al. (2016)), or a baseline of the GLM (e.g., Fujimoto et al. (2017)). The signal of interest can additionally be compared to a different channel not covering the region of interest (Liu et al., 2016) or asymmetry scores can be calculated, e.g., difference between right- and left-hemispheric channels (Aranyi et al., 2016). For the two studies using a multivariate approach, the feedback was based on the output of the classifier identifying neutral or positive affect (Trambaiolli et al. (2018), Weyand et al. (2015)). Two studies (Narita (2015), Kober et al. (2018)) did not report the methods used for feedback calculation. Using the system baseline instead of a preceding baseline before each trial seems risky, since low-frequency drifts may confound the signal if the applied preprocessing methods do not capture them properly.

Another important aspect is the selection of feedback thresholds, i.e., defining the amount of signal change necessary for change and setting a minimum and maximum of the presented feedback. For example, thresholds were defined based on signal variation during the preceding control condition (Aranyi et al., 2016; Kimmig et al., 2018) on the basis of t-values, which was also used for feedback (Fujimoto et al., 2017), or based on a certain HbO change (Kinoshita et al., 2016). Li et al. (2019) calibrated thresholds based on a pre-experiment conducted in an independent sample.

However, some studies did not transparently report how the threshold used for feedback was defined (see also Section 3.2). 


\subsection{Conclusion - Online signal-processing methods and hardware}

While the studies reviewed applied a considerable diversity of online signal-processing methods, some similarities across the studies were also evident, e.g., regarding online-feature and chromophore selection. Unfortunately, crucial information regarding online signal-processing procedures was often missing. Since there are no established standards for online or offline processing methods (Kamran et al., 2016; Pinti et al., 2018b), future studies are encouraged to explore different methods and provide sufficient information so that other studies can easily replicate successful methods.

Assuring signal quality is crucial for neurofeedback applications, particularly for fNIRSneurofeedback, which suffers from strong extracranial artifacts. A careful selection of online signalprocessing methods is necessary to avoid the presentation of invalid feedback information. Further developments and more systematic research on fNIRS online signal-processing methods are definitely needed to overcome this specific shortcoming of the fNIRS technology. 
Table 2. Online signal-processing methods and hardware.

\begin{tabular}{|c|c|c|c|c|c|c|c|}
\hline \multirow[t]{2}{*}{ Study } & \multirow{2}{*}{$\begin{array}{l}\text { Data for } \\
\text { Section } 2.1 \\
\text { Device }\end{array}$} & \multirow{2}{*}{$\begin{array}{l}\text { Data for Section } 2.2 \\
\begin{array}{l}\text { Selections of target regions (channel(s) of } \\
\text { interest) }\end{array}\end{array}$} & \multicolumn{2}{|c|}{ Data for Section 2.4} & \multicolumn{2}{|l|}{ Data for Section 2.3} & \multirow{2}{*}{$\begin{array}{l}\text { Data for Section } 2.5 \\
\text { Calculation of feedback signal }\end{array}$} \\
\hline & & & Online feature & $\begin{array}{l}\text { Chromo- } \\
\text { phore }\end{array}$ & Online preprocessing & $\begin{array}{l}\text { Artifact } \\
\text { control }\end{array}$ & \\
\hline $\begin{array}{l}\text { Aranyi et al. } \\
\text { (2016) }\end{array}$ & fNIR400 & 8 channels positioned on subject's forehead & $\begin{array}{l}\text { Asymmetry (left } \\
\text { vs. right dIPFC } \\
\text { amplitudes) }\end{array}$ & $\mathrm{HbO}$ & $\begin{array}{l}\text { Low-pass filter (finite impulse } \\
\text { response, order 20) } 0.1 \mathrm{~Hz} \text {, sliding- } \\
\text { window motion artifact rejection, } \\
\text { reference channel }\end{array}$ & $\begin{array}{l}\text { reference } \\
\text { channels }\end{array}$ & $\begin{array}{l}\text { Threshold of signal based on mean, SD, and signal } \\
\text { variation of signal during counting (baseline) }\end{array}$ \\
\hline $\begin{array}{l}\text { Barth et al. } \\
\text { (2016) }\end{array}$ & ETG-4000 & $\begin{array}{l}14 \text { channels aligned with positions of EEG } 10-20 \\
\text { system, registered to MNI space (Tsuzuki et al. } \\
\text { 2007) }\end{array}$ & Amplitude, PFC & $\mathrm{HbO}$ & Not reported & none & Change in $\mathrm{HbO}$ compared to $15 \mathrm{~s}$ baseline at beginning. \\
\hline $\begin{array}{l}\text { Fujimoto et al. } \\
\text { (2017) }\end{array}$ & OMM-3000 & $\begin{array}{l}4 \text { channels aligned with positions of EEG } 10-20 \\
\text { system. MNI positions estimated using individual } \\
\text { structural MRI and digitizer measurements }\end{array}$ & $\begin{array}{l}\text { Amplitude (t- } \\
\text { values } \\
\text { estimated by } \\
\text { GLM), SMA }\end{array}$ & $\mathrm{HbO}$ & $\begin{array}{l}\text { GLM analysis. } 20 \text { s sliding window. } \\
\text { Linear term to correct drift. } 4 \text { short- } \\
\text { distance channels (principal } \\
\text { component included as regressor in } \\
\text { GLM). }\end{array}$ & $\begin{array}{l}\text { EMG, } 4 \\
\text { short- } \\
\text { distance } \\
\text { channels }\end{array}$ & $\begin{array}{l}\text { Contrast regulation vs rest (adaptive GLM, } 20 \mathrm{~s} \text { sliding } \\
\text { window), maximum t-value from the } 4 \text { channels. Primary } \\
\text { principal component of short-distance channels as } \\
\text { nuisance regressor }\end{array}$ \\
\hline $\begin{array}{l}\text { Hosseini et al. } \\
\text { (2016) }\end{array}$ & ETG-4000 & $\begin{array}{l}\text { Channels aligned with positions of EEG 10-20 } \\
\text { system (Okamoto et al., 2004). Functional } \\
\text { localizer (working memory task) }\end{array}$ & $\begin{array}{l}\text { Amplitude, } \\
\text { dIPFC }\end{array}$ & $\mathrm{HbO}$ & Bandpass filter $0.01-0.5 \mathrm{~Hz}$ & no & $\begin{array}{l}\text { Change in the average } \mathrm{HbO} \text { signal over feedback channels } \\
\text { (over } 9 \mathrm{~s} \text { window) relative to the calibration period }\end{array}$ \\
\hline $\begin{array}{l}\text { Hudak et al. } \\
\text { (2017) }\end{array}$ & ETG-4000 & $\begin{array}{l}8 \text { channels aligned with positions of EEG 10-20 } \\
\text { system, registered to MNI space (Tsuzuki et al. } \\
\text { 2007) }\end{array}$ & $\begin{array}{l}\text { Amplitude, } \\
\text { dIPFC/IFG }\end{array}$ & $\mathrm{HbO}$ & $\begin{array}{l}\text { Kalman filter with a } 5 \mathrm{~s} \text { sliding } \\
\text { window, CAR of all channels }\end{array}$ & CAR & $\begin{array}{l}\text { Change in } \mathrm{HbO} \text { compared to } 5 \mathrm{~s} \text { baseline, CAR of all } \\
\text { channels subtracted }\end{array}$ \\
\hline $\begin{array}{l}\text { Hudak et al. } \\
\text { (2018) }\end{array}$ & ETG-4000 & $\begin{array}{l}8 \text { channels aligned with positions of EEG 10-20 } \\
\text { system, registered to MNI space (Tsuzuki et al. } \\
\text { 2007) }\end{array}$ & $\begin{array}{l}\text { Amplitude, } \\
\text { dIPFC/IFG }\end{array}$ & $\mathrm{HbO}$ & $\begin{array}{l}\text { Bandpass filter: } 0.01-1 \mathrm{~Hz}, 5 \mathrm{~s} \text { moving } \\
\text { average, CAR of all channels }\end{array}$ & CAR & $\begin{array}{l}\text { Change in } \mathrm{HbO} \text { compared to } 5 \mathrm{~s} \text { baseline, CAR of all } \\
\text { channels subtracted }\end{array}$ \\
\hline $\begin{array}{l}\text { Kanoh et al. } \\
\text { (2011) }\end{array}$ & ETG-4000 & $\begin{array}{l}3 \text { channels aligned with positions of EEG 10-20 } \\
\text { system }\end{array}$ & $\begin{array}{l}\text { Amplitude } \\
\text { sensorimotor } \\
\text { cortex }\end{array}$ & $\mathrm{HbO}$ & $\begin{array}{l}\text { High-pass filter and 7-point moving } \\
\text { average }\end{array}$ & none & $\begin{array}{l}\text { Average of channels / no baseline before task (not } \\
\text { explicitly reported) }\end{array}$ \\
\hline $\begin{array}{l}\text { Kimmig et al. } \\
\text { (2018) }\end{array}$ & ETG-4000 & $\begin{array}{l}10 \text { channels aligned with positions of EEG 10-20 } \\
\text { system, registered to MNI space (Tsuzuki et al. } \\
\text { 2014) }\end{array}$ & $\begin{array}{l}\text { Amplitude, } \\
\text { dIPFC/IFG }\end{array}$ & $\mathrm{HbO}$ & $\begin{array}{l}5 \mathrm{~s} \text { moving average filter, CAR of } \\
\text { remaining channels }\end{array}$ & CAR & $\begin{array}{l}\text { Average of channels, change in } \mathrm{HbO} \text { compared to } 5 \mathrm{~s} \\
\text { baseline. Standard deviation from previous trial used to } \\
\text { scale maximum/minimum of feedback signal. CAR of } \\
\text { remaining channels subtracted. }\end{array}$ \\
\hline $\begin{array}{l}\text { Kinoshita et al. } \\
\text { (2016) }\end{array}$ & ETG-4000 & $\begin{array}{l}6 \text { channels aligned with positions of EEG 10-20- } \\
\text { system, registered to MNI space (Tsuzuki et al. } \\
\text { 2007). }\end{array}$ & $\begin{array}{l}\text { Amplitude, } \\
\text { frontopolar } \\
\text { cortex }\end{array}$ & $\mathrm{HbO}$ & Not reported & $\begin{array}{l}\text { respiratory } \\
\text { rate }\end{array}$ & $\begin{array}{l}\text { Average of channels, moving baseline, last } 10 \mathrm{~s} \text {. } \\
\text { Maximum display of the bar graph was }+0.25[\mathrm{mMmm}]\end{array}$ \\
\hline $\begin{array}{l}\text { Kober et al. } \\
\text { (2014) }\end{array}$ & ETG-4000 & $\begin{array}{l}8 \text { channels aligned with positions of EEG 10-20 } \\
\text { system registered to MNI space (Singh et al., } \\
\text { 2005). Functional localizer to select channels } \\
\text { with best signal quality during a motor task }\end{array}$ & $\begin{array}{l}\text { Asymmetry } \\
\text { (difference } \\
\text { between left } \\
\text { and right motor } \\
\text { area) }\end{array}$ & $\mathrm{HbO}$ & $\begin{array}{l}0.01 \mathrm{HPF} \text { and } 1.5 \mathrm{~Hz} \text { LPF, } 2 \mathrm{~s} \text { moving } \\
\text { average. Difference of right and left } \\
\text { channels (also cancels out artifacts) }\end{array}$ & $\begin{array}{l}4 \text { reference } \\
\text { channels }\end{array}$ & $\mathrm{HbO}$ left vs. $\mathrm{HbO}$ right. No baseline period before trials \\
\hline $\begin{array}{l}\text { Kober et al. } \\
\text { (2015) }\end{array}$ & ETG-4000 & $\begin{array}{l}4 \text { channels aligned with positions of EEG } 10-20 \\
\text { system, positions assessed via individual digitizer } \\
\text { measurements }\end{array}$ & $\begin{array}{l}\text { Amplitude } \\
\text { (difference } \\
\text { between IFG } \\
\text { and posterior } \\
\text { regions) }\end{array}$ & $\begin{array}{l}\mathrm{HbO} \text { and } \mathrm{HbR} \\
\text { group }\end{array}$ & $\begin{array}{l}0.01 \text { high- and } 1.5 \mathrm{~Hz} \text { low-pass filter, } 2 \\
\mathrm{~s} \text { moving average. Difference of IFG } \\
\text { and of posterior reference channels }\end{array}$ & $\begin{array}{l}\text { EMG, } 4 \\
\text { reference } \\
\text { channels }\end{array}$ & $\begin{array}{l}\mathrm{HbO} / \mathrm{HbR} \text { of } \mathrm{FB} \text { channels vs reference channels. No } \\
\text { baseline period before trials }\end{array}$ \\
\hline $\begin{array}{l}\text { Kober et al. } \\
\text { (2018) }\end{array}$ & NIRSport & $\begin{array}{l}4 \text { channels, positions assessed via individual } \\
\text { digitizer measurements, probably based on EEG } \\
10-20 \text { system }\end{array}$ & Amplitude, IFG & $\begin{array}{l}\mathrm{HbO} \text { and } \mathrm{HbR} \\
\text { group }\end{array}$ & Not reported & none & Not reported \\
\hline $\begin{array}{l}\text { Lapborisuth et } \\
\text { al. (2017) }\end{array}$ & LABNIRS & $\begin{array}{l}14 \text { channels aligned with positions of EEG } 10-20 \\
\text { system, positions assessed via individual digitizer } \\
\text { measurements }\end{array}$ & $\begin{array}{l}\text { Amplitude, } \\
\text { motor cortex }\end{array}$ & $\mathrm{HbO}$ & $\begin{array}{l}\text { Detrending and normalization (last } 10 \\
\text { s) }\end{array}$ & none & $\begin{array}{l}\text { Difference of current sample divided by SD of previous } 10 \\
s \text { and linear trend of previous } 10 \mathrm{~s}\end{array}$ \\
\hline
\end{tabular}


fNIRS-Neurofeedback - a systematic review

Table 2. (continued)

\begin{tabular}{|c|c|c|c|c|c|c|c|}
\hline \multirow[t]{2}{*}{ Study } & \multirow{2}{*}{\begin{tabular}{|l|} 
Data for \\
Section 2.1 \\
Device
\end{tabular}} & \multirow{2}{*}{$\begin{array}{l}\text { Data for Section } 2.2 \\
\text { COI selection }\end{array}$} & \multicolumn{2}{|c|}{ Data for Section 2.4} & \multicolumn{2}{|l|}{ Data for Section 2.3} & \multirow{2}{*}{$\begin{array}{l}\text { Data for Section } 2.5 \\
\text { Calculation of feedback signal }\end{array}$} \\
\hline & & & Online feature & $\begin{array}{l}\text { Chromo- } \\
\text { phore }\end{array}$ & Online preprocessing & $\begin{array}{l}\text { Artifact } \\
\text { control }\end{array}$ & \\
\hline $\begin{array}{l}\text { Lee et al. } \\
\text { (2015) }\end{array}$ & FOIRE-3000 & $\begin{array}{l}7 \text { channels aligned with positions of EEG 10-20 } \\
\text { system. Positions assessed via individual digitizer } \\
\text { measurements }\end{array}$ & $\begin{array}{l}\text { Amplitude, } \\
\text { sensorimotor } \\
\text { cortex }\end{array}$ & $\mathrm{HbO}$ & Not reported & none & t-values, probably GLM \\
\hline Li et al. (2019) & NIRSport & $\begin{array}{l}1 \text { channel aligned with positions of EEG } 10-20 \\
\text { system. Position of channel validated by MRI } \\
\text { scans in two independent participants }\end{array}$ & Amplitude, OFC & $\mathrm{HbO}$ & $2 \mathrm{~s}$ moving average & none & $\begin{array}{l}\text { Change in HbO compared to } 2 \mathrm{~s} \text { baseline, feedback scaled } \\
\text { based on a pre-experiment ("difficulty coefficient") }\end{array}$ \\
\hline Liu et al. (2016) & ETG-4000 & $\begin{array}{l}\text { Functional localizer at beginning of each session } \\
\text { (channel with highest/lowest signal during face } \\
\text { processing), positions assessed via individual } \\
\text { digitizer measurements }\end{array}$ & $\begin{array}{l}\text { Amplitude, } \\
\text { frontal and } \\
\text { temporal face } \\
\text { processing } \\
\text { regions }\end{array}$ & $\mathrm{HbO}$ & $\begin{array}{l}\text { Reference channel (channel irrelevant } \\
\text { for face-processing network identified } \\
\text { during functional localizer) }\end{array}$ & $\begin{array}{l}\text { reference } \\
\text { channel }\end{array}$ & $\begin{array}{l}\text { HbO Percent signal change in feedback channel } \\
\text { compared to reference channel (face matching compared } \\
\text { to house matching) }\end{array}$ \\
\hline $\begin{array}{l}\text { Marx et al. } \\
\text { (2015) }\end{array}$ & ETG-4000 & $\begin{array}{l}8 \text { channels aligned with positions of EEG 10-20 } \\
\text { system, registered to MNI space (Tsuzuki et al. } \\
\text { 2007) }\end{array}$ & $\begin{array}{l}\text { Amplitude, } \\
\text { dIPFC/IIFG }\end{array}$ & $\mathrm{HbO}$ & Common average reference (CAR) & no & Change in $\mathrm{HbO}$ compared to $5 \mathrm{~s}$ baseline \\
\hline $\begin{array}{l}\text { Mihara et al. } \\
\text { (2012) }\end{array}$ & OMM-3000 & $\begin{array}{l}3 \text { channels aligned with positions of EEG } 10-20 \\
\text { system, positions estimated using structural MRI } \\
\text { and digitizer measurements of representative } \\
\text { participants }\end{array}$ & $\begin{array}{l}\text { Amplitude (t- } \\
\text { values } \\
\text { estimated by } \\
\text { GLM), premotor } \\
\text { cortex }\end{array}$ & $\mathrm{HbO}$ & $\begin{array}{l}\text { GLM analysis. } 20 \mathrm{~s} \text { sliding window, } \\
\text { linear term to correct for drift. } \\
\text { Autoregressive model order } 1 \text { to } \\
\text { adjust autocorrelation, excluded } 3 \\
\text { participants with finger movement }\end{array}$ & $\begin{array}{l}\text { no, but } \\
\text { reported } \\
\text { offline } \\
\text { control }\end{array}$ & $\begin{array}{l}\text { Contrast regulation vs rest (adaptive GLM, } 20 \mathrm{~s} \text { sliding } \\
\text { window), maximum t-value from the } 3 \text { channels }\end{array}$ \\
\hline $\begin{array}{l}\text { Mihara et al. } \\
\text { (2013) }\end{array}$ & OMM-3000 & $\begin{array}{l}3 \text { channels aligned with positions of EEG } 10-20 \\
\text { system, positions estimated using individual MRI } \\
\text { and digitizer measurements }\end{array}$ & $\begin{array}{l}\text { Amplitude (t- } \\
\text { values } \\
\text { estimated by } \\
\text { GLM), premotor } \\
\text { cortex }\end{array}$ & $\mathrm{HbO}$ & $\begin{array}{l}\text { GLM analysis, } 20 \text { s sliding window. } \\
\text { Linear term to correct for drift, } \\
\text { autoregressive model order } 1 \text { to } \\
\text { adjust autocorrelation, EMG control }\end{array}$ & EMG & $\begin{array}{l}\text { Contrast regulation vs rest (adaptive GLM, } 20 \mathrm{~s} \text { sliding } \\
\text { window), maximum t-value from the } 3 \text { channels }\end{array}$ \\
\hline Narita (2015) & $\begin{array}{l}\text { PocketNIRS, } \\
\text { Dynasense }\end{array}$ & $\begin{array}{l}1 \text { channel aligned with positions of EEG 10-20 } \\
\text { system. No information about registration } \\
\text { reported. }\end{array}$ & Amplitude, PFC & $\mathrm{HbO}$ & Not reported & none & Not reported \\
\hline $\begin{array}{l}\text { Trambaiolli et } \\
\text { al. (2018) }\end{array}$ & NIRScout & $\begin{array}{l}32 \text { channels aligned with positions of EEG 10-20 } \\
\text { system. No information about registration } \\
\text { reported }\end{array}$ & $\begin{array}{l}\text { Output of } \\
\text { classifier, } \\
\text { frontal/occipital } \\
\text { networks }\end{array}$ & $\mathrm{HbO}, \mathrm{HbR}$ & $\begin{array}{l}1 \mathrm{~Hz} \text { low-pass filter and } 3 \mathrm{~s} \text { moving } \\
\text { average, normalization by average } \\
\text { signal from the same channel during } \\
\text { previous neutral condition }\end{array}$ & none & $\begin{array}{l}\text { Output of linear discriminant classifier based on } \mathrm{HbO} \text { and } \\
\mathrm{HbR} \text { of } 32 \text { channels, indicating neutral or positive affect }\end{array}$ \\
\hline $\begin{array}{l}\text { Weyand et al. } \\
\text { (2015) }\end{array}$ & Imagent & $\begin{array}{l}9 \text { channels aligned with positions of EEG 10-20 } \\
\text { system. No information about registration } \\
\text { reported }\end{array}$ & Classifier, PFC & $\begin{array}{l}\mathrm{HbO}, \mathrm{HbR}, \\
\mathrm{tHb}\end{array}$ & $\begin{array}{l}\text { Third-order Chebyshev IIR cascade } \\
\text { filter with pass-band edge frequency } \\
\text { of } 0.1 \mathrm{~Hz} \text {, stop-band edge frequency } \\
\text { of } 0.5 \mathrm{~Hz} \text {, and pass band ripple of } 0.1 \\
\mathrm{~dB}\end{array}$ & none & $\begin{array}{l}\text { Session 1-5: weighted slope score. Session 6-16: output } \\
\text { of classifier. LDA classifier using } 8 \text { features selected from } \\
288 \text { different temporal and spatiotemporal features from } \\
\text { all channels and chromophores }\end{array}$ \\
\hline
\end{tabular}

cortex; tHb total hemoglobin. 


\section{Quality of published studies}

In this section, we assess and discuss the quality of published studies including (1) features of experimental designs and methodological quality according to the JBI ratings, (2) design and reporting quality according to the CRED-nf checklist, and (3) analysis of statistical power/sensitivity as an indicator of reliability of the reported findings.

\subsection{Quality of experimental designs}

Table 3.1 shows important features of the experimental designs of the studies.

\subsubsection{Control conditions}

Depending on the specific research aim, neurofeedback studies can make use of several different control conditions. Control conditions may include, treatment-as-usual, bidirectional-regulation control, feedback of an alternative brain signal, sham feedback, and mental-rehearsal control, and can be applied in a within- or between-subject design (see Sorger et al., 2019). Ideally, multiple control conditions are applied in order to disentangle neurofeedback-specific from unspecific processes. In this regard, Lubianiker et al. (2019) recently proposed an extension of established control conditions, in which participants of the control group are randomly assigned to a subset of different neural control targets (randomized ROI control condition). In this way, specific effects related to the control targets and neurofeedback-unspecific processes that likely differ for different neurofeedback targets may average out across all subjects of this control group. For extensive discussions on different control conditions in neurofeedback research, see Lubianiker et al., (2019) and Sorger et al., (2019).

Seven of the studies did not use any control condition. Nine studies used a sham feedback based on either artificially created signals (five studies) or based on a brain signal of another participant (yoked feedback, four studies). Two studies compared the effects with other forms of biofeedback (EEG- or EMG-based). One study compared the effects of motor-imagery neurofeedback with mental rehearsal (motor imagery only) and another compared neurofeedback during a motor task with motor task only in a within-subject design. Also, the effects of neurofeedback based on $\mathrm{HbO}$ and $\mathrm{HbR}$ were compared in a between-subject design without an additional sham-feedback condition (Kober et al., 2015) and in a bidirectional-control approach, investigating four different groups (Kober et al., 2018).

\subsubsection{Randomization and blinding}

Of the fifteen studies with a control group, eleven studies randomized assignment to groups or order of conditions. Seven studies blinded participants to conditions and only one applied double-blinding. However, in some situations blinding is not possible, e.g., when comparing neurofeedback to another treatment. These studies attempted to reduce bias by not informing participants about any other treatment than that which they received (Hudak et al., 2017; Marx et al., 2015), which may at least help to keep levels of expectation and motivation equal across groups. Assessments of motivation, expectation, or other unspecific factors can be included in future studies to check this assumption.

\subsubsection{Assessment of transfer}

To assess a transfer effect, four studies made use of transfer trials or no-feedback conditions, where participants received the same instructions as in the neurofeedback task, but without receiving any neurofeedback information. As described in Section 1.4, some studies used a very liberal definition of the term transfer (if at all a very near transfer; see Barnett and Ceci, 2002). Five studies (Barth et 
al., 2016; Hudak et al., 2017; Kimmig et al., 2018; Kober et al., 2015; Mihara et al., 2012) assessed transfer using other computerized tasks and investigated whether activation within the targeted brain region changes after neurofeedback training, and were able to demonstrate transfer beyond neurofeedback training (far transfer).

\subsubsection{Follow-up measures}

Only four of the studies investigated long-term effects of neurofeedback in a follow-up (ten days up to six months after neurofeedback training) in order to investigate whether participants were still able to regulate brain activity after a period without training (Weyand et al., 2015) or stability of observed behavioral effects (Marx et al., 2015). Evidence of delayed effects of neurofeedback emerging after the primary endpoint of a study has been reported. Particularly when investigating clinical populations, follow-up measures may boost statistical power and should be applied where possible (Rance et al., 2018; Van Doren et al., 2019).

\subsubsection{Methodological quality (JBI critical appraisal tool)}

To assess the methodological quality of the included studies, we used the checklist for quasiexperimental studies of the Joanna Briggs Institute (JBI) critical appraisal tools (Tufanaru et al., 2017). Two of the authors (SK and DM) independently rated studies according to the nine criteria of the checklist. These items include: clarity of cause and effect (temporal relationship between variables), similar participants; similar treatment in compared groups; existence of a control group/condition; multiple measurement points of the outcome; completion of follow-up; similar outcome measurements in compared groups; reliability of outcome measurements; appropriate statistical methods. Each study was allocated points based on the number of criteria fulfilled. Disagreements between the review authors were resolved by discussion. For further details about rating criteria, see Supplementary Material 2.

Table S1 shows the results of the ratings for each study. On average 5.55 (SD =2.15) of 9 required quality criteria were rated "yes"., It should be noted that only four studies used appropriate statistical methods according to this rating. Most of the studies were rated as not using appropriate statistical methods because they did not justify their sampling plan or omitted labelling their study a pilot, feasibility or proof-of-concept study. 


\section{Table 3.1. Study designs.}

\begin{tabular}{|c|c|c|c|c|c|c|c|}
\hline \multirow[t]{2}{*}{ Study } & \multirow{2}{*}{$\begin{array}{l}\text { Data for } 1.2 \text { and Fig 1B } \\
\text { Target }\end{array}$} & \multirow{2}{*}{$\begin{array}{l}\text { Data for } 1.2 \text { and Fig } 1 \mathrm{~A} \\
\text { Participants }\end{array}$} & \multicolumn{5}{|l|}{ Data for 3.1} \\
\hline & & & Control group & Randomization & Blinding & Follow up & Transfer \\
\hline Aranyi et al. (2016) & dIPFC asymmetry & 18 healthy & None & no & no & no & no \\
\hline Barth et al. (2016) & PFC & 13 healthy & None & no & no & no & separate task \\
\hline $\begin{array}{l}\text { Fujimoto et al. } \\
\text { (2017) }\end{array}$ & SMA & 20 healthy & sham (yoked feedback, within) & yes & single-blinded & no & no \\
\hline Hosseini et al. (2016) & dIPFC & 20 healthy & sham (yoked feedback) & no & not reported & no & no \\
\hline Hudak et al. (2017) & bilateral dIPFC/IFG & 20 highly impulsive & EMG biofeedback & yes & no & no & separate tasks \\
\hline $\begin{array}{l}\text { Hudak et al. (2018) - } \\
\text { excerpt from Mayer } \\
\text { et al. (2015) }\end{array}$ & bilateral dIPFC/IFG & 19 adults with $A D H D$ & None & no & no & $\begin{array}{l}\text { not reported, } \\
\text { but protocol } \\
\text { included FU }\end{array}$ & no \\
\hline Kanoh et al. (2011) & left sensorimotor cortex & 5 healthy & None & no & no & no & separate task \\
\hline Kimmig et al. (2018) & bilateral dIPFC/IFG & 12 SAD & None & no & no & no & separate task \\
\hline $\begin{array}{l}\text { Kinoshita et al. } \\
\text { (2016) }\end{array}$ & $\begin{array}{l}\text { bilateral frontal pole } \\
\text { cortex }\end{array}$ & 24 healthy & sham feedback (artificially generated, within) & yes & single-blinded & no & yes \\
\hline Kober et al. (2014) & motor cortex asymmetry & 17 healthy & sham (yoked feedback, within) & yes & single-blinded & no & no \\
\hline Kober et al. (2015) & bilateral IFG & 20 healthy & HbO vs. HbR-group & yes & single-blinded & no & separate task \\
\hline Kober et al. (2018) & bilateral IFG & $\begin{array}{l}48 \text { healthy / } 12 \text { per } \\
\text { group }\end{array}$ & bidirectional control for $\mathrm{HbO}$ and $\mathrm{HbR}$ & yes & single-blinded & no & no \\
\hline $\begin{array}{l}\text { Lapborisuth et al. } \\
\text { (2017) }\end{array}$ & left motor cortex & 22 healthy & motor imagery without feedback (within) & no & no & no & yes \\
\hline Lee et al. (2015) & $\begin{array}{l}\text { sensory motor cortex } \\
\text { (S1,M1, SMA) }\end{array}$ & 4 healthy & motor task without feedback (within) & unclear & no & no & no \\
\hline Li et al. (2019) & right lateral OFC & 60 healthy & sham (yoked feedback) & yes & single-blinded & no & no \\
\hline Liu et al. (2016) & $\begin{array}{l}\text { frontal and temporal face } \\
\text { processing regions }\end{array}$ & 2 healthy, 2 ASD & sham feedback (artificially generated) & yes & not reported & no & no \\
\hline Marx et al. (2015) & bilateral dIPFC/IFG & $\begin{array}{l}27 \text { children ADHD / } 9 \\
\text { per group }\end{array}$ & EEG and EMG biofeedback & no & no & $\begin{array}{l}2 \text { weeks and } 6 \\
\text { months }\end{array}$ & separate tasks \\
\hline Mihara et al. (2012) & left premotor cortex & 21 healthy & sham feedback (artificially generated, within) & yes & single-blinded & no & no \\
\hline Mihara et al. (2013) & $\begin{array}{l}\text { ipsilesional premotor } \\
\text { cortex }\end{array}$ & 20 stroke patients & sham feedback (artificially generated) & yes & double-blinded & 2 weeks & no \\
\hline Narita (2015) & left PFC & 4 ASD & None & no & no & $1-3$ months & no \\
\hline $\begin{array}{l}\text { Trambaiolli et al. } \\
(2018)\end{array}$ & $\begin{array}{l}\text { frontal and occipital } \\
\text { networks }\end{array}$ & 33 healthy & sham feedback (artificially generated, within) & $\begin{array}{l}\text { conditions presented } \\
\text { in random order }\end{array}$ & $\begin{array}{l}\text { single-blinded? Not } \\
\text { clearly reported }\end{array}$ & no & no \\
\hline Weyand et al. (2015) & bilateral PFC & 10 healthy & None & no & no & 10 days & no \\
\hline
\end{tabular}

ADHD, attention-deficit/hyperactivity disorder; ASD, autism spectrum disorder; ADHD dIPFC, dorsolateral prefrontal cortex; EEG, electroencephalography; EMG, electromyography; IFG, inferior frontal gyrus; HbR, deoxyhemoglobin; OFC, orbitofrontal cortex; HbO, oxyhemoglobin; PFC, prefrontal cortex; SAD, social anxiety disorder; SMA, supplementary motor are 


\subsection{Experimental design and reporting quality (CRED-nf checklist)}

To assess experimental design and reporting quality we used the current version of the CRED-nf (Consensus on the reporting and experimental design of clinical and cognitive-behavioral neurofeedback studies) checklist (Ros et al., 2020). The CRED-nf checklist is designed to encourage best practice in terms of experimental designs and reporting of neurofeedback studies. It covers seven domains (Pre-experiment, Control groups, Control measures, Feedback specifications, Outcome measures brain, Outcome measures behavior, and Data storage), including 23 checklist items, fifteen of which are considered essential, and eight encouraged. In contrast to the JBI checklist, the CRED-nf checklist does not include subjective ratings. Instead, it assesses whether a study reports contents required by a respective item, e.g., "Report the feedback modality and content". One of the authors (SK) filled in a checklist for each study with page numbers identifying where each point was addressed. The number of addressed items of both categories essential and encouraged are reported for each study. For further details see Supplementary Material 3.

Table 3.2 and Table S2 show detailed results of the CRED-nf checklist, including a short description of individual items of the respective CRED-nf domains. On average $63.03 \%(\sim 9$ of 15$)$ of the essential items and $10.23 \%(\sim 1$ of 8$)$ of encouraged items were reported. It should be noted that this best-practice checklist was published only very recently after all the included studies had been published. Hence, the authors of the included studies as well as ongoing studies could not make use of this resource in designing their experiments and publishing their results.

The domain Pre-experiment was not very well reported, on average only $20.45 \%$ of the items were included. Only one study reported a pre-registration of the experimental protocol (Hudak et al., 2018). However, this study deviated from the originally published registration (Mayer et al., 2015) and investigated a different research question including only a subsample of participants. None of the studies conducted a power analysis in order to justify sample size. Eight of the 22 studies were labeled as a pilot, feasibility, or proof-of-concept study, which renders a power analysis unnecessary.

On average only $25.45 \%$ of the items in the domain Control groups were reported, which is related to the fact that only a few clinical trials have yet been published and seven uncontrolled studies were included. Of the fifteen controlled studies, nine reported having used single- or doubleblinding or at least discussed the fact that blinding was not possible (Hudak et al., 2017; Marx et al., 2015). Only one study reported having blinded raters of the outcomes or whether participants/experimenters remained blinded. Two of the clinical studies employed a standard-of-care intervention group as a benchmark for improvement (Marx et al., 2015; Mihara et al., 2013).

With respect to the domain Control measures, the studies reported on average $42.73 \%$ of the items. Six studies reported having used some measure of psychosocial or nonspecific factors (e.g., motivation, expectation, effort). Since these measures are easy to implement and do not require much additional time, we recommend that future studies should make more use of this additional easy and low-cost method of controlling for nonspecific effects. Almost a third of the studies did not report whether participants were provided with a strategy and only six studies reported strategies used by the participants. Even if no explicit strategies are provided, study design and general instructions about the experiment may prime the use of certain strategies. The use of mental strategies undoubtedly affects brain activity and may also induce behavioral effects on its own. Hence, better control and transparent and more detailed reporting of this factor is required, which may also contribute to solving outstanding issues of the utility of strategy instructions (see also 1.4.1). 
The domain Feedback specifications was well reported, on average $90 \%$ were included. All studies included feedback modality and content as well as the software and hardware used, and almost all studies reported the definition of the online-feature extraction. Sixteen of the studies also included at least some information about the reinforcement schedule, for example feedback-threshold criteria, but did not justify this in relation to the existing literature. Also, the amount of reward received by participants was rarely included. While most studies reported the essential contrast used for feedback, i.e., regulation $v s$. rest, only eight of the studies described both conditions of regulation and rest separately.

Studies reported on average $53.03 \%$ of the items related to Outcome measures - Brain. Most studies included neurofeedback regulation success based on the feedback signal. However, some studies did not report regulation success at all (Marx et al., 2015; Narita, 2015), while others only reported regulation success of representative participants or only based on significant channels and took surrounding channels that were not used for feedback calculation into account instead of reporting the average of all feedback channels (e.g., Lapborisuth et al., 2017; Mihara et al., 2012). This lack of transparency makes it difficult to conclude whether neurofeedback was at all effective on the brain level. Less than half of the studies plotted the feedback signal of within-session or betweensession regulation blocks of the feedback signal, which would permit further insights into the dynamics of regulation performance over the course of the training period. Only seven of the studies statistically compared the experimental to the control condition or group. There were seven uncontrolled studies unable to make such a comparison. Hence, seven controlled studies did not undertake this comparison, but rather analyzed groups/conditions separately, which does not allow any conclusions to be drawn about group differences and makes it difficult to appraise the effectiveness of neurofeedback (Nieuwenhuis et al., 2011).

On average only $18.18 \%$ of items in the domain Outcome measures - Behavior were reported. However, this low rate can be explained by the fact that most studies were mainly interested in regulation performance, and behavioral variables did not represent the primary outcome or were not assessed at all. Of the 14 studies that actually assessed a behavioral variable, eight reported a correlation between regulation success and behavioral outcome. None of the studies included measures of clinical or behavioral significance, such as the minimal clinically important difference (MCID; Engel et al., 2018). However, there are only a few clinical studies in which such a measure is applicable.

None of the studies reported having uploaded materials, analysis scripts, code, or data to an open-access data repository.

\subsubsection{CRED-nf checklist - Conclusion}

In sum, the reporting quality of published studies can be considered to be moderate to low in the light of current consensus guidelines. While some domains such as Feedback specifications were well reported, other such as Pre-experiment or Data Storage were not. The CRED-nf checklist provides guidelines for best practice with regard to experimental design and reporting of neurofeedback studies. The field of fNIRS-neurofeedback is still very young and some of the standards set by the CRED-nf checklist do not yet apply at this early stage. For example, double-blinding or a measure of clinical or behavioral significance should be included at the stage of randomized controlled trials, but not necessarily in early proof-of-concept studies that primarily aim to test neural regulation performance. However, we note that we applied more lenient criteria for some of the items, which resulted in higher ratings than if we had applied the original items. This should be considered in future comparisons applying the CRED-nf checklist. As stated above, this best-practice checklist was 
not available when the studies included in this review were published. Hence, a lack of reporting for some items should be expected at this early stage. Nevertheless, the incomplete reporting identified in the domain Outcome measures - Brain gives reason for concern. Without this information, we cannot clearly identify whether participants learned to control the brain signal of interest. As is common across the published literature, findings that do not meet a threshold for statistical significance may remain underreported in the fNIRS-neurofeedback literature. Future studies could benefit from orienting towards these new guidelines, while also abiding by other guidelines for reporting clinical trials, such as CONSORT (Schulz et al., 2010). We encourage researchers to use the CRED-nf checklist as an orientation when designing new studies and suggest they make use of the CRED-nf web application (rtfin.org/CREDnf) when submitting results for publication. The web application helps authors to standardize the reporting of CRED-nf items, which will facilitate future systematic reviews.

\section{Table 3.2. Reporting and design quality according to the CRED-nf checklist.}

\begin{tabular}{lc} 
CRED-nf domain & M \pm SD \\
\hline Pre-experiment & $20.45 \pm 25.16 \%$ \\
Control groups & $25.45 \pm 22.41 \%$ \\
Control measures & $42.73 \pm 22.51 \%$ \\
$\begin{array}{l}\text { Feedback } \\
\text { specifications }\end{array}$ & $90.00 \pm 13.45 \%$ \\
$\begin{array}{l}\text { Outcome measures - } \\
\text { brain }\end{array}$ & $53.03 \pm 31.97 \%$ \\
$\begin{array}{l}\text { Outcome measures - } \\
\text { behavior }\end{array}$ & $18.18 \pm 24.62 \%$ \\
Data storage & $0.00 \pm 0.00 \%$ \\
CRED-nf essential & $63.03 \pm 18.49 \%$ \\
CRED-nf encouraged & $10.23 \pm 9.94 \%$ \\
CRED-nf total & $44.66 \pm 13.56 \%$ \\
\hline
\end{tabular}

Mean percentages and standard deviations of items rated "yes" for the different domains of the CRED-nf checklist. Detailed results for individual studies and items, including a description of the items of the respective domains, can be found in Table S2. CRED-nf, consensus on the reporting and experimental design of clinical and cognitive-behavioral neurofeedback studies checklist (Ros et al., 2020).

\subsection{Statistical power/sensitivity}

To further assess study quality, we extracted information about the reported sample sizes and estimated statistical power/sensitivity of the included studies. Statistical power, the probability of detecting an effect size of a certain magnitude with a given sample size and accepted threshold for the probability of a false-positive finding, is an important indicator for the reliability of reported findings and will therefore be investigated here.

The majority of neuroscientific studies, including neuroimaging studies, feature relatively small sample sizes and hence remarkably low statistical power for detecting realistic (i.e., small to moderate) effect sizes (Button et al., 2013; Poldrack et al., 2017). Furthermore, small sample sizes imply higher variability around effect size estimates. In combination with publication bias (i.e., the tendency to publish mainly significant findings), reported effects thus tend to be overestimated 
(Algermissen and Mehler, 2018), rendering the scientific literature in psychology and neuroscience an unreliable basis for conducting power analyses for future studies (Allen and Mehler, 2019; Schäfer and Schwarz, 2019; Szucs and Ioannidis, 2017).

Computing statistical power requires knowledge of the effect size that is considered relevant and worth detecting, i.e., the smallest effect size of interest (SESOI). Post hoc power analyses that are commonly not based on SESOIs represent mere transformations of the p-value. Such an approach cannot therefore provide any information about the a priori power of the included fNIRS studies. Since SESOIs are highly experiment-specific (Lakens et al., 2018) and unknown to us, it proved difficult to set an SESOI for 1) regulation performance and 2) behavioral outcomes for our purpose. First, we note that neurofeedback experiments represent complex interventions (Sitaram et al., 2017) during which various factors, or their interaction, may determine the outcome (see also Section 1.4). We also note that neurofeedback studies vary greatly in how they define and quantify successful regulation (Paret et al., 2019) and different definitions may reveal different effect sizes. Second, SESOIs for behavioral outcomes will depend on the specific paradigm (e.g., motor imagery or emotion regulation), outcome variable (e.g., reaction times or self-rated measures of emotion regulation), and study population (i.e., healthy participants or patient populations, and for patient groups the type of clinical population, e.g., stroke or depressed patients). Therefore, we did not set an SESOI to calculate the post hoc statistical power of fNIRS-neurofeedback studies, but instead used Cohen's conventions, covering a range of effect sizes that may be comparable to potential SESOIs (d=0.2, 0.5 and 0.8; (Cohen, 1992)).

Additionally, we assessed the statistical sensitivity, calculating the smallest effect size that individual studies were able to detect with certain probabilities ( 0.8 and 0.95$)$, given their reported sample size. We conducted separate analyses for regulation performance and for a behavioral outcome, where applicable. Several studies employed a repeated-measure analysis of variance (ANOVA) but did not provide details of the correlation among repeated measures or violation of the sphericity assumption. For pragmatic reasons, we thus assumed for all studies that there was no violation of sphericity and a correlation of 0.8 , which is considered a good general estimate for testretest correlations in neuropsychological assessments (Calamia et al., 2013). To simplify analyses, we also used uncorrected p-values and ANOVA/t-tests instead of non-parametric tests, if used by a specific study. Additionally, instead of mixed models or ANOVAs with more than one within factor, two-factorial mixed ANOVAs were used for the sensitivity analysis. Overall, these measures should lead to higher power estimation, and we can assume that we overestimated the statistical power of the studies. Analyses were carried out using G*Power (Faul et al., 2007)

Overall, sample sizes varied across studies from our minimum inclusion size, i.e. four (two per cell), up to 60 (30 per cell). One single group study included 33 participants. The median sample size of the studies was 20 (12 per cell). We calculated the median effect size that was detectable with $80 \%$ and $95 \%$ power. For the outcome regulation performance, we found a value of $d=0.75$ and $d=1$, respectively. For behavioral outcomes, this was value was $d=0.52$ and $d=0.69$, respectively. We further found that the median power to detect a small effect of $\mathrm{d}=0.2$ was low ( 0.16 and 0.22 ). Our results showed that the power was only sufficient to detect large effects of $d=0.8$ regulation performance and 0.97 for behavioral effects, respectively (see Table 3.3 and Tables S3-4 for more details). Figure 3.3 shows power curves for detecting different effect sizes with a sample size of 20 participants, which is equivalent to the median sample size of the studies included in this review, for different commonly used statistical tests. 
Some studies that included control groups lacked a direct statistical comparison of regulation performance between the experimental and the control condition. Instead, these studies only reported the main effects within conditions and compared statistical significance between conditions instead of effect sizes. This statistical approach is erroneous for group comparisons (Nieuwenhuis et al., 2011) and makes it difficult to assess whether the experimental group outperformed the control group. If we assume that these studies found no statistically significant group effect, we note that due to insufficient statistical power we cannot come to valid conclusions about potential group effects. To check this assumption, we conducted sensitivity analyses for the respective group/interaction effects, using a statistical test that was appropriate for the respective study design (see Supplementary Material 5). While sensitivity for detecting a certain group/interaction effect size remained the same, it is reasonable to assume that interaction effects are smaller and, depending on the underlying assumptions, require four to sixteen times more participants to achieve similar a priori statistical power (Gelman, 2018). Therefore, studies were likely underpowered for reliably detecting group differences in neurofeedback effects, which are very likely smaller than within-effects.

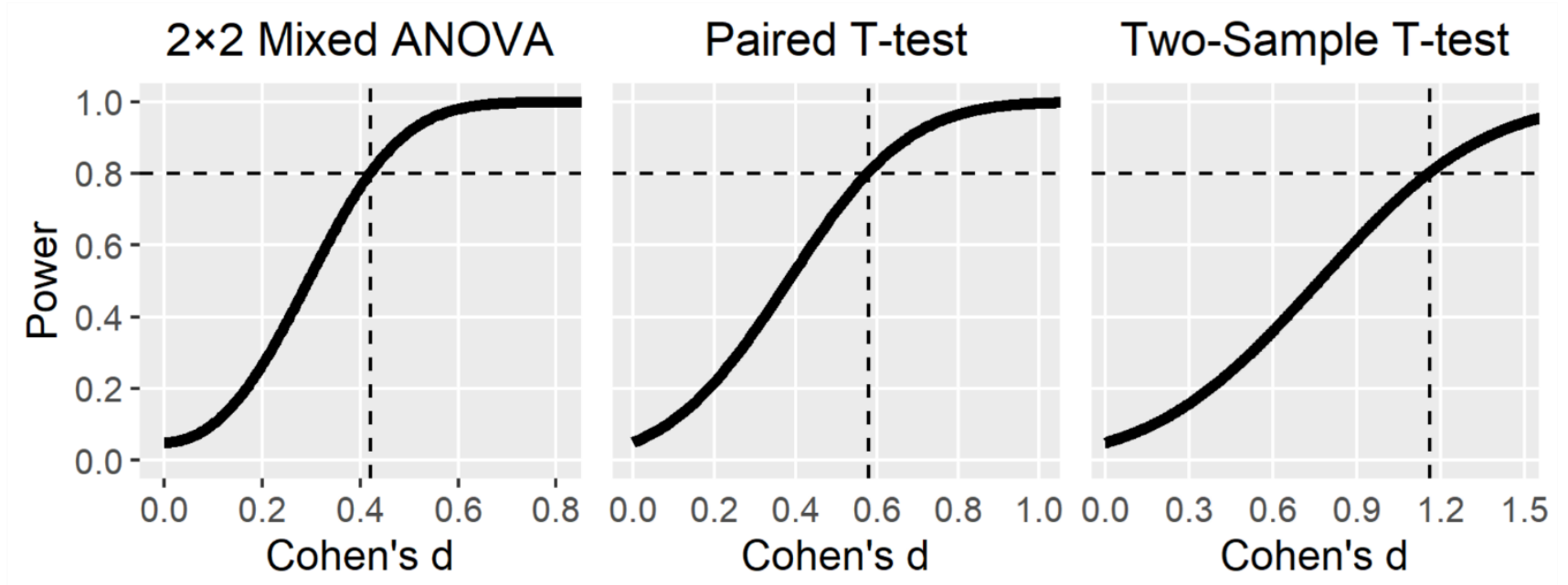

Figure 3.3. Statistical power curves to detect different effect sizes with 20 participants (median sample size) for different statistical tests. Dashed lines indicate smallest effect sizes detectable with $80 \%$ power. Note that the power curve for the $2 \times 2$ mixed ANOVA was based on liberal statistical assumptions (e.g., high correlation among repeated measures, sphericity and uncorrected p-value of $0.05)$.

As noted earlier, it remains difficult, if not impossible, to generalize these findings across paradigms because the smallest relevant effect sizes (SESOIs) may depend on the choice of the neurofeedback target region, population, control conditions, and other characteristics of the design. However, specific behavioral effects of neurofeedback, as assessed in placebo and motivation levelcontrolled designs, are possibly rather small (see Supplementary Material 5). This assumption is at least partly supported for the EEG-neurofeedback literature where well-controlled studies depending on population and rating report no specific group effect (e.g., Schabus et al., 2017; Schönenberg et al., 2017) or medium to small and non-significant effect sizes (Strehl et al., 2017).

Altogether, our results suggest the median sample size of published fNIRS-neurofeedback studies is relatively small at $\mathrm{N}=20$, which is comparable to sample sizes reported more broadly for neuroimaging (Poldrack et al., 2017). It is thus not surprising that most studies lack sufficient statistical sensitivity to detect (realistic) SESOIs. In contrast, most studies were biased towards finding only relatively large effects (Figure 3.3). We further note that the analyses we present are 
based on rather liberal statistical assumptions (e.g., no application of multiple-testing correction) and thus likely still overestimate the true statistical sensitivity and power in the field.

Particularly with regard to specific behavioral effects, studies still lack statistical sensitivity and thus allow only very limited conclusions to be drawn about the feasibility of the paradigms and no conclusions about the specificity of fNIRS-neurofeedback effects. The field mainly consists of small feasibility, pilot, or proof-of-concept studies, which do not have to fulfill the same requirements with regard to sample size since their purpose is to explore the potential of fNIRSneurofeedback in self-regulating a target brain signal and modulating behavior. However, as described above, most studies are not sufficiently transparent about this and hence risk overstating their findings. We thus reiterate previous concerns and recommend that authors appropriately label pilot, feasibility, and proof-of-concept studies, ideally in their manuscript title. Furthermore, we recommend a clear distinction between planned and exploratory analyses.

Table 3.3. Sensitivity and statistical power for reported analysis.

\begin{tabular}{|c|c|c|c|c|c|c|}
\hline \multicolumn{7}{|c|}{ Regulation performance } \\
\hline \multirow[b]{2}{*}{ Study } & \multirow[b]{2}{*}{$\mathbf{N}$} & \multicolumn{2}{|c|}{ Sensitivity } & \multicolumn{3}{|c|}{ Power to detect } \\
\hline & & $80 \%$ power & $95 \%$ power & $d=0.2$ & $d=0.5$ & $d=0.8$ \\
\hline Mean & 22.11 & $d=0.88$ & $d=1.15$ & 0.20 & 0.55 & 0.74 \\
\hline Median & 20 & $d=0.75$ & $d=1$ & 0.16 & 0.48 & 0.8 \\
\hline \multicolumn{7}{|c|}{ Behavioral outcomes } \\
\hline \multirow{2}{*}{ Study } & \multirow{2}{*}{$\mathbf{N}$} & \multicolumn{2}{|c|}{ Sensitivity } & \multicolumn{3}{|c|}{ Power to detect } \\
\hline & & $80 \%$ power & 95\% power & $d=0.2$ & $d=0.5$ & $d=0.8$ \\
\hline Mean & 22.1 & $d=0.66$ & $d=0.87$ & 0.20 & 0.68 & 0.87 \\
\hline Median & 20 & $d=0.53$ & $d=0.69$ & 0.22 & 0.76 & 0.97 \\
\hline
\end{tabular}

Note that in order to simplify the analysis for some studies, we performed the analysis for a different statistical test than originally reported, did not take into account correction for multiple comparisons, and assumed no sphericity violation and a high correlation among repeated measures for ANOVAs. Overall, these measures should have led to an overestimation of the statistical power/sensitivity of the studies.

\subsection{Conclusion - Quality of published studies}

Altogether, the design and reporting quality of the studies can be considered to be moderate. There were a few studies of lower quality, but also some high-quality studies including sham-control conditions, randomization, and blinding (Figure 3). Sample sizes of the studies were small and thus their statistical power to detect realistic effects was low. While similar results have been reported for other fields within the neurosciences (Button et al., 2013; Nieuwenhuis et al., 2011; Szucs and Ioannidis, 2017), fNIRS-neurofeedback is still in its infancy and we thus still have the chance to tackle these issues early on and lay a more robust foundation to build upon in future work. As the field moves on, well-designed, sufficiently powered confirmatory studies are necessary to reach valid conclusions about the effectiveness of fNIRS-neurofeedback. 


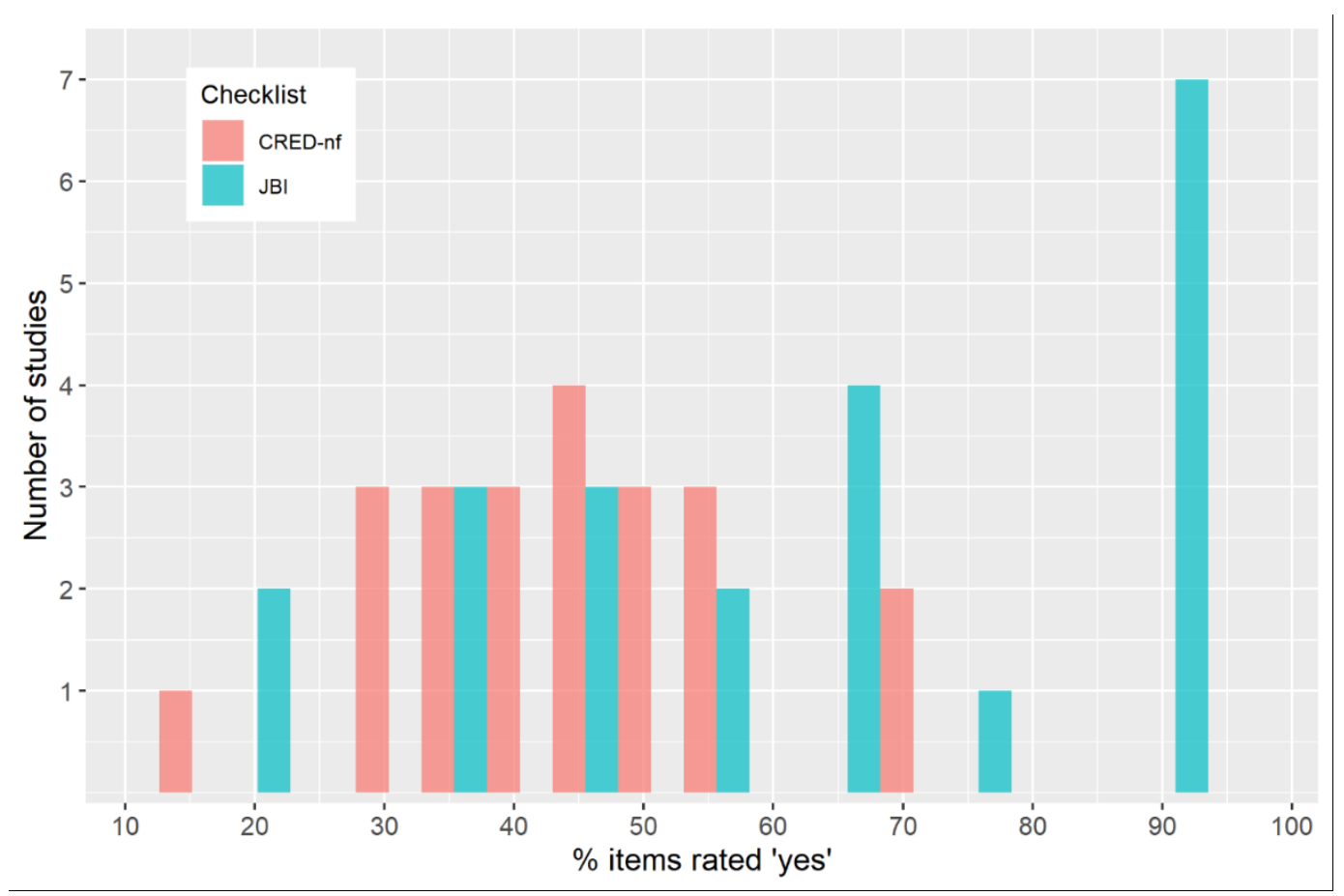

Figure 3. Quality of studies according to the CRED-nf and JBI checklist.

\section{Neural effects of neurofeedback}

In this section, we assess and discuss the effectiveness of fNIRS-neurofeedback for regulating and inducing pre-post changes in brain activity.

\subsection{Neurofeedback regulation success}

Before considering training effects in terms of changes in behavior or brain activity after neurofeedback, an essential question is whether neurofeedback training was successful, meaning was it effective for regulating the target brain signal. Unfortunately, no standard has been established so far, and there are a number of different ways to define and quantify neurofeedback regulation success. Paret et al., (2019) provide a taxonomy and discuss this issue in depth. In this section, we first discuss different success measures as applied by the studies and then systematically assess and critically discuss the effectiveness of fNIRS-neurofeedback for regulating a target brain signal, as reported by the studies (see Table 4.1).

\subsubsection{Neurofeedback regulation success - Measures}

While most fNIRS-neurofeedback studies define regulation success based on the magnitude of a signal change, some also define it on the basis of time, i.e., on the amount of time in a trial during which the feedback signal exceeds a defined threshold (Hudak et al., 2017, 2018; Kimmig et al., 2018). Most studies apply a fixed threshold approach and compare the regulation statistically to a baseline condition over a session, while some also report success rates, i.e., a ratio of successful (as previously defined) trials per session (Aranyi et al., 2016; Trambaiolli et al., 2018; Weyand et al., 2015) or for a group of sessions (Hudak et al., 2017, 2018; Kimmig et al., 2018). None of the studies calculated personal effect sizes, i.e., divided average signal change by the standard deviation of a session or run to account for individual noise (cf. Paret et al., 2019). Finally, to judge the regulation success of neurofeedback training, effects over time should be assessed for potential learning curves, rather than merely assessing average signal change across all trials compared to the baseline. For 
instance, comparisons can be made between early (first session(s)/trial(s)) and late parts of the training period (last sessions(s)/trial(s)) (Fujimoto et al., 2017; Kimmig et al., 2018; Kober et al., 2014). Alternatively, assuming linear improvement, regulation success can be assessed over all trials or sessions via linear regression (Kober et al., 2018; Li et al., 2019). Ultimately, success measures should be compared with a control group or within control conditions. This is important since also sham feedback induces activations, particularly in frontal brain regions (Ninaus et al., 2013), which is the target of the majority of fNIRS-neurofeedback studies. Certain studies compared some of the first sessions with some of the last sessions (Kimmig et al., 2018; Kober et al., 2014), which seems arbitrary. If certain sessions or trials are selected for comparison, this should be theoretically or empirically justified. For example Fujimoto et al. (2017) compared the first six trials with the last ten trials, because according to the existing literature participants should reach a plateau after the first six trials during a motor-learning task (Hatakenaka et al., 2007).

It should be noted that, with a few exceptions in fMRI-neurofeedback (e.g., Goldway et al., 2019), variability measures have been neglected in most neurofeedback success definitions. According to learning theories, the probability of a certain behavior (brain activity) should increase after learning (Skinner, 1963), but variability (noise) should also be reduced. Some theorists have compared neurofeedback learning to motor skill learning (Sitaram et al., 2017). From this perspective, it is assumed that in the course of learning a new skill variability is reduced to optimize performance. (He et al., 2016).

\subsubsection{Neurofeedback regulation success - Results}

We assessed whether the studies reported an effect for each of the aforementioned comparisons (see Table 4.1 and Figure 4.1). Overall, results show that fNIRS-neurofeedback can be used to regulate brain activity, with some of the studies also demonstrating a greater increase over time as compared to a control group (Fujimoto et al., 2017; Kober et al., 2014; Li et al., 2019; Mihara et al., 2013). Particularly these studies were all of higher quality according to our ratings, at least single- or double-blinded and applied a sham-feedback approach (see Section 3.1). The other studies reported mixed results, lacked a control group, or did not report results sufficiently well, making it difficult to draw definite conclusions. For example, some studies did not report regulation success based on the feedback signal, but rather results from an offline analysis of all channels, and did not clarify whether significant effects overlap with channels used for feedback (e.g., Lapborisuth et al., 2017; Mihara et al., 2012). This impedes any judgement about the success of a neurofeedback protocol. We encourage authors to report regulation success based on all feedback channels. If more than one channel is used, the average of the channels or an ROI analysis only based on feedback channels can be reported. Furthermore, some of the studies used time-based binary success criteria and did not additionally report brain activation during regulation. These criteria require the definition of a threshold to be surpassed and neglect information about the signal amplitude. Some studies (Hudak et al., 2017, 2018; Kimmig et al., 2018) used a threshold of zero, i.e., spent at least half of the time of the last $15 \mathrm{~s}$ of a trial in the desired direction. This threshold is very liberal and it can be expected that random fluctuation (only noise) of a signal should be half of the time above and half of the time below zero. Hence, high success rates can be expected by chance and are not informative. When using time-based criteria, the amplitudes of the feedback signal should be reported as well. 


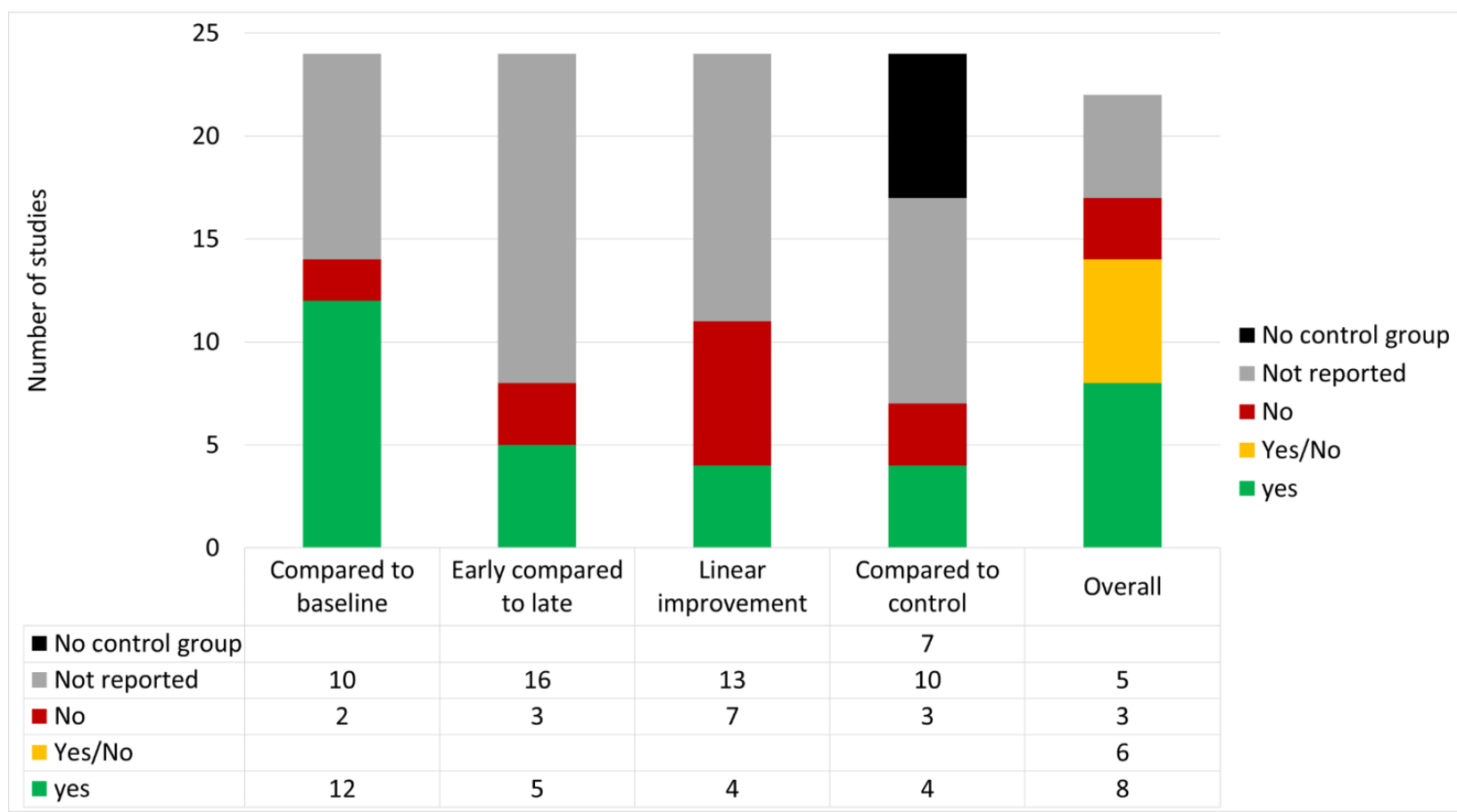

Figure 4.1. Neurofeedback regulation success. Overall regulation was classified "Yes" if a significant effect for one or more of the four measures were reported, and "No" if no significant effect was reported. If both were reported overall regulation was classified "Yes/No". Note that Kober et al., $(2015,2018)$ trained regulation of $\mathrm{O} 2 \mathrm{Hb}$ and $\mathrm{HHb}$ in different groups and found differential results for the groups. Therefore, the two studies were counted twice for the four measures. In overal regulation both studies were only vounted once and classified as "Yes/No".

\subsection{Neural changes over time and neural mechanisms}

In addition to analyzing regulation success, some studies explored pre-post changes in neural outcomes or investigated neural mechanisms of neurofeedback as assessed during training. Hudak et al., (2017) found increased activation of the left dlPFC (part of the region trained) during NoGo trials after neurofeedback compared to an active control group, but there was also a baseline difference between groups, and decreased activity in the experimental group before neurofeedback training may explain the effect. No effect was reported for the working-memory task, which is in line with (Barth et al., 2016), who reported only marginal decreases for frontal and language-related brain regions during a working-memory task. Kimmig et al. (2018) found no change in social-threat processingrelated brain activity after neurofeedback, but a change in the right inferior parietal sulcus, right inferior frontal gyrus, and supplementary motor cortex correlated positively with a change in social anxiety. Mihara et al. (2012) found increased activation of the left premotor cortex in channels not used for feedback, and decreased activity in the parietal association cortex, which was related to an increase in the sham-feedback group, speculatively related to switching to visual imagery strategies due to the incorrect feedback in this group. Kober et al. (2015) demonstrated transfer of neurofeedback training and showed that activity within the IFG during motor imagery and motor execution of swallowing decreased (HbR increased) after neurofeedback training.

Some studies analyzed brain connectivity during neurofeedback training. Hudak et al. (2018) identified differential brain connectivity patterns for successful and failed regulation of the dlPFC, and demonstrated the importance of the fronto-parietal control network. Results may be specific to the training protocol and online analysis (i.e., influence of reference channels punishing activation in 
certain brain regions). However, Trambaiolli et al. (2018) found similar connectivity patterns for the random-, real- and fixed-feedback (i.e., no-feedback) condition during an affective neurofeedback task.

\subsection{Conclusion - Neural effects of neurofeedback}

All in all, the results of some high-quality studies demonstrate the effectiveness of fNIRS-

neurofeedback for regulating brain activation in motor regions (three of the successful studies) and in the OFC (one study). For other brain regions, such as the dlPFC, results are mixed and we cannot conclude whether fNIRS-neurofeedback is effective for regulating these regions as well. Moreover, a lacuna in reporting regulation success is evident as well, which, together with a high level of degrees of freedom on the part of the researchers as outlined above, raises suspicions of selectively reporting positive results (Simmons et al., 2011) and inflated effect sizes (Ioannidis, 2008). Furthermore, initial analyses provide preliminary evidence for potential neuroplastic effects of fNIRS-neurofeedback and further mechanistic insights. Future studies should follow up on such efforts and investigate neural mechanisms of neurofeedback. Neuroplastic effects may also be investigated in combination with other methods such as fMRI, which has higher spatial resolution and covers subcortical brain regions that contribute to neurofeedback learning (Emmert et al., 2016; Sitaram et al., 2017). 
Table 4.1. Neurofeedback regulation success

\begin{tabular}{|c|c|c|c|c|c|c|c|}
\hline \multirow[t]{3}{*}{ Study } & \multirow{3}{*}{$\begin{array}{l}\text { Data for } 1.2 \text { and } \\
\text { Fig } 1 \mathrm{~B} \\
\text { Target region }\end{array}$} & \multirow{3}{*}{$\begin{array}{l}\text { Data for } 1.2 \text { and } \\
\text { Fig } 1 \mathrm{~A} \\
\text { Target population }\end{array}$} & \multirow{3}{*}{$\begin{array}{l}\text { Data for } 4.1 \\
\text { Success measure and analysis }\end{array}$} & \multicolumn{4}{|c|}{ Data for 4.1 and Fig. 4.1} \\
\hline & & & & \multicolumn{4}{|c|}{ Successful regulation as compared to } \\
\hline & & & & CTB & ECTL & Linear & CTC \\
\hline $\begin{array}{l}\text { Aranyi et al. } \\
(2016)\end{array}$ & dIPFC asymmetry & 18 healthy & $\begin{array}{l}\text { Success rate. Successful trial = statistically significant increase in average } \\
\text { asymmetry during NF compared to counting baseline ( } \mathrm{t} \text {-test) }\end{array}$ & yes & NR & no & $\mathrm{N} / \mathrm{A}$ \\
\hline $\begin{array}{l}\text { Barth et al. } \\
\text { (2016) }\end{array}$ & PFC & 13 healthy & Fixed threshold. Average increase of $\mathrm{HbO}$ over whole training course & yes & NR & NR & $\mathrm{N} / \mathrm{A}$ \\
\hline $\begin{array}{l}\text { Fujimoto et } \\
\text { al. (2017) }\end{array}$ & SMA & 20 healthy & $\begin{array}{l}\text { Fixed threshold. Significant increase in } \mathrm{HbO} \text { during late compared to early } \\
\text { trials (time/condition interaction) }\end{array}$ & yes & yes & NR & yes \\
\hline $\begin{array}{l}\text { Hosseini et } \\
\text { al. (2016) }\end{array}$ & dIPFC & 20 healthy & $\begin{array}{l}\text { Fixed threshold. Linear regression over trials within and over sessions. } \\
\text { Further group analysis offline (but overlap with feedback channels } \\
\text { unclear) }\end{array}$ & no & no & no $^{1}$ & $\mathrm{NR}^{2}$ \\
\hline $\begin{array}{l}\text { Hudak et al. } \\
\text { (2017) }\end{array}$ & $\begin{array}{l}\text { bilateral } \\
\text { dIPFC/IFG }\end{array}$ & $\begin{array}{l}20 \text { highly } \\
\text { impulsive }\end{array}$ & $\begin{array}{l}\text { Success rate. Successful trial = at least } 7 \mathrm{~s} \text { of the last } 15 \mathrm{~s} \text { regulation in the } \\
\text { desired direction. Average of all trials from first four sessions compared to } \\
\text { last four sessions }\end{array}$ & NR & no & no & NR \\
\hline $\begin{array}{l}\text { Hudak et al. } \\
\text { (2018) - }\end{array}$ & $\begin{array}{l}\text { bilateral } \\
\text { dIPFC/IFG }\end{array}$ & $\begin{array}{l}19 \text { adults with } \\
\text { ADHD }\end{array}$ & $\begin{array}{l}\text { Success rate. Successful trial = at least } 7 \mathrm{~s} \text { of the last } 15 \mathrm{~s} \text { regulation in the } \\
\text { desired direction. Average of all trials from first half compared to last half } \\
\text { of sessions }\end{array}$ & yes & no & no & $\mathrm{N} / \mathrm{A}$ \\
\hline $\begin{array}{l}\text { Kanoh et al. } \\
\text { (2011) }\end{array}$ & $\begin{array}{l}\text { left sensorimotor } \\
\text { cortex }\end{array}$ & 5 healthy & Fixed threshold. Linear regression over sessions & NR & NR & no & $\mathrm{N} / \mathrm{A}$ \\
\hline $\begin{array}{l}\text { Kimmig et } \\
\text { al. (2018) }\end{array}$ & $\begin{array}{l}\text { bilateral } \\
\text { dIPFC/IFG }\end{array}$ & $12 \mathrm{SAD}$ & $\begin{array}{l}\text { Success rate. Successful trial = at least } 7 \mathrm{~s} \text { of the last } 15 \mathrm{~s} \text { regulation in the } \\
\text { desired direction. Last three sessions compared to first three sessions. }\end{array}$ & NR & yes & NR & N/A \\
\hline $\begin{array}{l}\text { Kinoshita et } \\
\text { al. (2016) }\end{array}$ & $\begin{array}{l}\text { bilateral frontal } \\
\text { pole cortex }\end{array}$ & 24 healthy & $\begin{array}{l}\text { Fixed threshold. Increased } \mathrm{HbO} \text { in feedback channels compared to rest } \\
\text { baseline }\end{array}$ & yes & NR & NR & no \\
\hline $\begin{array}{l}\text { Kober et al. } \\
(2014)\end{array}$ & $\begin{array}{l}\text { motor cortex } \\
\text { asymmetry }\end{array}$ & 17 healthy & Fixed threshold. Last three sessions vs first three sessions & yes & yes & yes & yes \\
\hline $\begin{array}{l}\text { Kober et al. } \\
(2015)\end{array}$ & bilateral IFG & 20 healthy & $\begin{array}{l}\text { Fixed threshold. } \mathrm{HbR} \text { increased over sessions in } \mathrm{HbR} \text { group and } \mathrm{HbO} \\
\text { decreased. In } \mathrm{HbO} \text { group } \mathrm{HbR} \text { increased over session as well. }\end{array}$ & yes $/$ no $^{3}$ & NR & yes $/ \mathrm{no}^{3}$ & NR \\
\hline $\begin{array}{l}\text { Kober et al. } \\
(2018)\end{array}$ & bilateral IFG & $\begin{array}{l}48 \text { healthy / } 12 \\
\text { per group }\end{array}$ & $\begin{array}{l}\text { Fixed threshold. Not compared to baseline, motor imagery and no group } \\
\text { comparison }\end{array}$ & NR & NR & yes $/ \mathrm{no}^{3}$ & NR \\
\hline
\end{tabular}


fNIRS-Neurofeedback - a systematic review

Table 4.1. (continued)

\begin{tabular}{|c|c|c|c|c|c|c|c|}
\hline \multirow[t]{3}{*}{ Study } & \multirow{3}{*}{$\begin{array}{l}\text { Data for } 1.2 \text { and } \\
\text { Fig } 1 \mathrm{~B} \\
\text { Target region }\end{array}$} & \multirow{3}{*}{$\begin{array}{l}\text { Data for } 1.2 \text { and } \\
\text { Fig } 1 \mathrm{~A} \\
\text { Target population }\end{array}$} & \multirow{3}{*}{$\begin{array}{l}\text { Data for } 4.1 \\
\text { Success measure and analysis }\end{array}$} & \multicolumn{4}{|c|}{ Data for 4.1 and Fig. 4.1} \\
\hline & & & & \multicolumn{4}{|c|}{ Successful regulation as compared to } \\
\hline & & & & CTB & ECTL & Linear & CTC \\
\hline $\begin{array}{l}\text { Lapborisuth } \\
\text { et al. (2017) }\end{array}$ & left motor cortex & 22 healthy & $\begin{array}{l}\text { Fixed threshold. Not clearly reported. Defined ROI based on motor } \\
\text { execution task (overlap with feedback channels not clear) and analyzed } \\
\text { HbR instead of HbO. No comparison between conditions and no effect for } \\
\text { HbO reported }\end{array}$ & NR & NR & NR & NR \\
\hline $\begin{array}{l}\text { Lee et al. } \\
\text { (2015) }\end{array}$ & $\begin{array}{l}\text { sensory motor } \\
\text { cortex }\end{array}$ & 4 healthy & $\begin{array}{l}\text { Fixed threshold. Increased HbO in feedback channels. No statistical } \\
\text { comparison between feedback and no feedback condition. }\end{array}$ & NR & NR & NR & NR \\
\hline $\begin{array}{l}\text { Li et al. } \\
\text { (2019) }\end{array}$ & right lateral OFC & 60 healthy & $\begin{array}{l}\text { Fixed threshold. Significant increase in } \mathrm{HbO} \text { over NF runs (time } \times \text { group } \\
\text { interaction). Regional specificity also confirmed by exploratory analysis of } \\
\text { all channels }\end{array}$ & yes & yes & yes & yes \\
\hline $\begin{array}{l}\text { Liu et al. } \\
(2016)\end{array}$ & $\begin{array}{l}\text { frontal and } \\
\text { temporal face } \\
\text { processing } \\
\text { regions }\end{array}$ & 2 healthy, 2 ASD & not reported & NR & NR & NR & NR \\
\hline $\begin{array}{l}\text { Marx et al. } \\
(2015)\end{array}$ & $\begin{array}{l}\text { bilateral } \\
\text { dIPFC/IFG }\end{array}$ & $\begin{array}{l}27 \text { children ADHD } \\
\text { / } 9 \text { per group }\end{array}$ & not reported & NR & NR & NR & NR \\
\hline $\begin{array}{l}\text { Mihara et } \\
\text { al. (2012) }\end{array}$ & $\begin{array}{l}\text { left premotor } \\
\text { cortex }\end{array}$ & 21 healthy & $\begin{array}{l}\text { Fixed threshold. Increase in } \mathrm{HbO} \text { compared to baseline for only one of the } \\
\text { three feedback channels reported }\end{array}$ & yes & NR & NR & no \\
\hline $\begin{array}{l}\text { Mihara et } \\
\text { al. (2013) }\end{array}$ & $\begin{array}{l}\text { ipsilesional } \\
\text { premotor cortex }\end{array}$ & 20 stroke patients & $\begin{array}{l}\text { Fixed threshold. Increased activation in one of the three FB channels } \\
\text { compared to baseline. Timeline analysis and ROI analysis. Statistical } \\
\text { details about timeline analysis missing }\end{array}$ & yes & yes & NR & yes \\
\hline $\begin{array}{l}\text { Narita } \\
(2015)\end{array}$ & left PFC & 4 ASD & not reported & NR & NR & NR & N/A \\
\hline $\begin{array}{l}\text { Trambaiolli } \\
\text { et al. (2018) }\end{array}$ & $\begin{array}{l}\text { frontal and } \\
\text { occipital } \\
\text { networks }\end{array}$ & 33 healthy & $\begin{array}{l}\text { Success rate. Successful trial = classifier. Percentage of successful trials for } \\
\text { all conditions }\end{array}$ & Yes & NR & NR & no \\
\hline $\begin{array}{l}\text { Weyand et } \\
\text { al. (2015) }\end{array}$ & bilateral PFC & 10 healthy & $\begin{array}{l}\text { Success rate. Successful trial = classifier. Average classification accuracy } \\
\text { for each session }\end{array}$ & yes & NR & NR & N/A \\
\hline
\end{tabular}

ADHD, attention-deficit/hyperactivity disorder; ASD, autism spectrum disorder; CTB, compared to baseline; ECTL, early compared to late, trail(s); CTC, compared to control group or within control condition; dIPFC, dorsolateral prefrontal cortex; IFG, inferior frontal gyrus; HbR, deoxyhemoglobin; NF, neurofeedback; OFC, orbitofrontal cortex; HbO, oxyhemoglobin; PFC, prefrontal cortex; SAD, social anxiety disorder; SMA, supplementary motor area. ${ }^{1}$ Marginal effects were considered "no", only significant effects were considered "yes". ${ }^{2}$ Effects

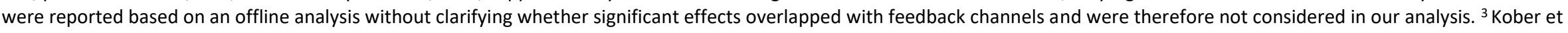
al. $(2015,2018)$ trained regulation of $\mathrm{HbO}$ and $\mathrm{HbR}$ in separate groups. 


\section{Behavioral effects of fNIRS-neurofeedback in healthy and clinical populations}

The ultimate goal of many neurofeedback applications is to induce significant effects in behavior as a prerequisite for developing clinical applications or neuroenhancing procedures. Because most studies have targeted prefrontal $(\mathrm{N}=13)$ or motor brain regions $(\mathrm{N}=7$; see Figure $1 \mathrm{~B})$, we here review the effectiveness for improving executive functioning and motor rehabilitation across patient and healthy populations (see Table 5.1).

\section{1. fNIRS-neurofeedback to improve executive functioning}

Hosseini et al., (2016) found mixed effects on working memory after task-based neurofeedback training targeting down-regulation of the dlPFC. Performance improved in an n-back task as compared to the sham-feedback group, but no improvement was found in either group for the delayed verbal working-memory task used for the task-based neurofeedback paradigm. Additionally, a positive effect on task switching (analyzed exploratory) was reported. It should be noted that this study only reported marginal effects for regulation performance. Unfortunately, Barth et al., (2016) did not report the behavioral effects for working memory. In a subclinical sample, Hudak et al. (2017) found no effect in an n-back task but improved inhibitory control as assessed with a Go-NoGo task, and only a trend compared to an EMG-biofeedback control group. Stop-signal reaction-time variability also decreased (significant group effect). The latter study also does not show any indications of successful regulation. Li et al., (2019) found a trend for enhanced cognitive flexibility as assessed with an attentional set-shifting task, but they did not apply a pre-measurement and compared groups only at post. Moreover, OFC regulation correlated with reward experience in the neurofeedback group only.

Due to its potential to improve prefrontal brain functions, particularly inhibitory control, fNIRS-neurofeedback has been investigated as a potential treatment for children (Marx et al., 2015) and adults (Hudak et al., 2018) with ADHD. Indeed, Marx et al., (2015) found indications of improved inhibitory control after neurofeedback in children with ADHD as assessed with a GoNoGo task, which was unfortunately not assessed in the control groups. Furthermore, this was accompanied by a decrease in ADHD symptoms, but similar improvements were also observed in the two active control groups. Therefore, while fNIRS-neurofeedback may improve attention and inhibitory control, leading to decreased ADHD symptoms, specificity has not been demonstrated yet and it remains open whether it offers any advantages over classical EEG-neurofeedback or other established treatments for ADHD. Larger clinical fNIRS-neurofeedback trials in children and adults with ADHD are currently under way (Blume et al., 2017; Mayer et al., 2015) and may shed further light on this issue.

Another potential clinical application for fNIRS-neurofeedback of the dlPFC is the treatment of social anxiety disorder. For example, it has been reported to reduce social threat-related attention bias and improve social and general trait anxiety as well as depressive symptoms (Kimmig et al., 2018). However, due to the absence of a control group, results are only preliminary. In addition, few indications of successful regulation were reported.

\section{2. fNIRS-neurofeedback for motor rehabilitation}

Two studies demonstrated the modulation of swallowing-related motor regions (within the IFG) in healthy participants. HbR increased during motor imagery and execution of swallowing after training, though not compared to a control group. This training protocol might be investigated in 
patients with dysphagia in the future (Kober et al., 2015, 2018). fNIRS-neurofeedback of premotor regions improved self-assessed kinesthetic motor imagery during real neurofeedback as compared to a within sham-feedback condition in healthy participants (Mihara et al., 2012), but did not improve postural stability after one session (Fujimoto et al., 2017). The significant interaction was mainly driven by a decrease in postural stability after sham feedback. This missing effect might be attributed to the limited duration of training or to ceiling effects in the healthy participants, as a longer training period in patients after stroke was efficacious (Mihara et al., 2013). In this double-blind randomized sham-controlled design, patients underwent stroke rehabilitation and were trained to upregulate activation of the ipsilesional premotor cortex via motor imagery. The control group also practiced motor imagery but received artificially generated feedback. After six sessions specific effects were observed for the hand/finger subscale of the Fugl-Meyer assessment. Results are promising and indicate that fNIRS-neurofeedback may facilitate motor recovery in patients after stroke, but replications in a larger, controlled clinical trial are needed.

\subsection{Other potential clinical applications}

FNIRS-neurofeedback was investigated as a potential treatment for autism spectrum disorder. Liu et al. (2016) applied a task-based neurofeedback approach to enhance the effects of facial-recognition training in adolescents with autism and found improved facial recognition which was also present in the patient receiving sham feedback. Given that these are only the initial data of a larger clinical trial which do not permit a statistical analysis, conclusions can be made only when all the data are published. Similarly, Narita (2015) trained four participants with autism to upregulate activity of the prefrontal cortex and reported improvements in working memory, inhibitory control as well as in anxiety and mood on a single-case level.

Furthermore, studies demonstrated successful classification of neutral and positive affective states (Trambaiolli et al., 2018) and regulation of asymmetric activation of the left dlPFC, a candidate neural mechanism of approach-avoidance motivation (Aranyi et al., 2016). These studies in healthy participants may pave the way for future applications in mood disorders.

Although not included in our review, it is worth mentioning a single-case study (Storchak et al., 2018) describing a new neurofeedback protocol to treat auditory verbal hallucinations in schizophrenia. A patient with paranoid schizophrenia was trained for 47 sessions to regulate the activity of the bilateral posterior superior temporal gyrus. To counteract neural correlates of auditory hallucinations, the patient was instructed to upregulate when expecting and downregulate when experiencing hallucinations. She was successful in upregulation, but not downregulation. However, even though amplitudes did not differ significantly from zero over the sessions, a learning effect was reported for downregulation, i.e., a significant decrease in activation over the sessions. Throughout the training period hallucinations decreased and symptoms improved.

\subsection{Conclusion - Behavioral effects of fNIRS-neurofeedback in healthy and clinical populations}

In sum, there is preliminary evidence for the effectiveness of fNIRS-neurofeedback for improving motor rehabilitation and executive functions, particularly for improved inhibitory control (Hudak et al., 2017; Marx et al., 2015) and cognitive flexibility (Hosseini et al., 2016; Li et al., 2019). Mixed results were reported for working-memory tasks, which may be attributable to ceiling effects (Hudak et al., 2017). This is in line with the EEG-neurofeedback literature where similar effects were observed for inhibitory control tasks (Bluschke et al., 2016; Mayer et al., 2016). However, nonspecific factors (psychosocial/placebo effects) may explain a large proportion of the effect sizes found in neurofeedback studies (Ros et al., 2020; Schönenberg et al., 2017; Thibault and Raz, 2016). 
Hence, due to the limited evidence available and a lack of well-powered (see Section 3.3) properly sham-controlled studies, it remains difficult to make any claims about the specificity of the reported effects. Regarding clinical potential, early pilot studies show the feasibility of fNIRS-neurofeedback in different patient populations such as ADHD, social anxiety disorder, autism, and stroke. The most promising data are found for stroke rehabilitation, where a double-blind sham-controlled study demonstrated beneficial effects (Mihara et al., 2013).It should be noted that most studies investigated effects in healthy populations and stronger effects may be expected in patient populations, displaying more room for improvement. Great optimism has been expressed with regard to future clinical applications (Ehlis et al., 2018), but larger well-controlled studies and clinical trials are needed to corroborate initial findings and demonstrate specificity before fNIRS-neurofeedback can be considered a viable complementary or even alternative treatment option. 


\section{Table 5.1. Behavioral effects of fNIRS-neurofeedback.}

\begin{tabular}{|c|c|c|c|}
\hline Study & Target region & $\begin{array}{l}\text { Target } \\
\text { population }\end{array}$ & Behavioral, cognitive/emotional effects \\
\hline Aranyi et al. (2016) & $\begin{array}{l}\text { dIPFC } \\
\text { asymmetry }\end{array}$ & 18 healthy & $\begin{array}{l}\text { Alignment ratings (the subjective rating of how appropriate the virtual agent's facial expressions were to the subject's thoughts during NF) } \\
\text { correlated with regulation success (within) }\end{array}$ \\
\hline $\begin{array}{l}\text { Fujimoto et al. } \\
\text { (2017) }\end{array}$ & SMA & 20 healthy & $\begin{array}{l}\text { Significant time } \times \text { group interaction for postural control, but no time effects for both groups-interaction was mainly driven by decreased } \\
\text { performance in the sham feedback group }\end{array}$ \\
\hline $\begin{array}{l}\text { Hosseini et al. } \\
\text { (2016) }\end{array}$ & dIPFC & 20 healthy & Improved working memory (significant time $\times$ group interaction). Exploratory: improved task switching \\
\hline Hudak et al. (2017) & $\begin{array}{l}\text { bilateral } \\
\text { dIPFC/IFG }\end{array}$ & $\begin{array}{l}20 \text { highly } \\
\text { impulsive }\end{array}$ & $\begin{array}{l}\text { Reduction in false alarms (Go-NoGo task, within effect, only trend for interaction). Reduction in stop-signal reaction times (SSRT) variability } \\
\text { (between effect), but no effect on SSRTs }\end{array}$ \\
\hline Kimmig et al. (2018) & $\begin{array}{l}\text { bilateral } \\
\text { dIPFC/IFG }\end{array}$ & 12 SAD & $\begin{array}{l}\text { Decreased social and general trait anxiety as well as depressive symptoms and a reduced disturbance of daily life. Improved social threat- } \\
\text { processing, i.e. reduction of social threat-related attention bias towards laughter, but not specifically for taunting vs. joyful laughter. No } \\
\text { correlation of behavioral effects with regulation performance }\end{array}$ \\
\hline Li et al. (2019) & $\begin{array}{l}\text { right lateral } \\
\text { OFC }\end{array}$ & 60 healthy & $\begin{array}{l}\text { Trend for enhanced cognitive flexibility (but only group comparison at post). Shorter response times and higher rewarding experience were } \\
\text { associated with stronger training-induced HbO increases in IOFC }\end{array}$ \\
\hline Liu et al. (2016) & $\begin{array}{l}\text { frontal and } \\
\text { temporal face } \\
\text { processing } \\
\text { regions }\end{array}$ & $\begin{array}{l}2 \text { healthy, } 2 \\
\text { ASD }\end{array}$ & Improved facial recognition in all participants (single case analysis, no statistic reported) \\
\hline Marx et al. (2015) & $\begin{array}{l}\text { bilateral } \\
\text { dIPFC/IFG }\end{array}$ & $\begin{array}{l}27 \text { children } \\
\text { ADHD / } 9 \text { per } \\
\text { group }\end{array}$ & $\begin{array}{l}\text { Decreased ADHD scores (parent and teacher ratings) within group. No effect on associated behavioral symptoms (SDQ) and quality of life (child } \\
\text { ratings). Go-NoGo RTs, RT variability and commission errors decreased within group. But RT effect only from post to follow up. TAP flexibility RTs } \\
\text { and RT variability decreased within group. In the control groups, ADHD scores did not significantly decrease, but also no significant group effect } \\
\text { was found. Baseline differences in quality of life and associated behavioral symptoms }\end{array}$ \\
\hline Mihara et al. (2012) & $\begin{array}{l}\text { left premotor } \\
\text { cortex }\end{array}$ & 21 healthy & Increased self-assessed kinesthetic motor imagery scores \\
\hline Mihara et al. (2013) & $\begin{array}{l}\text { ipsilesional } \\
\text { premotor } \\
\text { cortex }\end{array}$ & $\begin{array}{l}20 \text { stroke } \\
\text { patients }\end{array}$ & $\begin{array}{l}\text { Improved recovery of sensorimotor function, as assessed by Fugl-Meyer assessment scale (significant time } \times \text { group Interaction), no significant } \\
\text { adverse effect }\end{array}$ \\
\hline Narita (2015) & left PFC & 4 ASD & Improved working memory, performance in Stroop task, anxiety and mood (within-effect, only means, no statistic reported) \\
\hline $\begin{array}{l}\text { Weyand et al. } \\
\text { (2015) }\end{array}$ & bilateral PFC & 10 healthy & $\begin{array}{l}\text { Marginal decrease in task load comparing session } 10 \text { (strategy use) and } 15 \text { (voluntary self-regulation). Work load decreased and ease-of-use and } \\
\text { perceived intuitiveness only increased over self-regulation sessions (11-15), not over mental task sessions (1-10) }\end{array}$ \\
\hline
\end{tabular}

ADHD, attention-deficit/hyperactivity disorder; ASD, autism spectrum disorder; dIPFC, dorsolateral prefrontal cortex; IFG, inferior frontal gyrus; OFC, orbitofrontal cortex; PFC, prefrontal cortex; SAD, social anxiety disorder; SMA, supplementary motor area. 


\section{The potential of fNIRS for neurofeedback research - Future directions}

Neurofeedback research using fNIRS has just begun. Being more precise in targeting localized brain regions than EEG and much easier to use, and less expensive than fMRI, fNIRS may become an important tool for neurofeedback research and application. In this section, we outline our perspective on the future of fNIRS-neurofeedback and highlight its future potential.

Future research could benefit from exploiting the advantages of fNIRS to an even greater extent, since it offers unique opportunities for neurofeedback research. Compared to fMRI, it is easier to conduct a greater number of sessions and/or recruit more participants. This will help to solve the issue of low statistical power, which is a common problem in neuroscientific research (see Section 3.3.4). Furthermore, this makes this technique more suitable than fMRI for larger multicenter studies. To our knowledge, no clinical multicenter fMRI-neurofeedback study has been published, while in EEG-neurofeedback research it has already been demonstrated that multicenter studies are possible (e.g., Strehl et al., 2017). Methods applied in studies are still quite heterogeneous and further agreements and standardization of protocols are necessary before this step can be taken. If the target is a region of the neocortex, it is also conceivable that methods may be combined, and successful fMRI-neurofeedback protocols transferred to fNIRS-neurofeedback to conduct a high-powered study employing many sessions and/or participants. Future studies could also compare fNIRS-based with fMRI-based neurofeedback protocols using simultaneous measures to reveal commonalities and differences or even combine both methods to exploit optimally their advantages. For example, in a first fMRI session the regions of interest could be precisely defined on the individual level to guide the placement of optodes (channels of interest) and improve the spatial specificity for the following (more economic) fNIRS-neurofeedback sessions.

FNIRS is particularly suited for and has been extensively and successfully used in developmental neuroscience (Lloyd-Fox et al., 2010; Pinti et al., 2018b). Future research may benefit from using fNIRS-neurofeedback particularly in children and older healthy and patient populations. These populations show more movement during experiments (e.g. Poldrack et al., 2002) and such movements are more tolerable with fNIRS. They also possess physical features that may be beneficial for fNIRS signal quality (e.g., skull thickness, hair thickness/pigmentation; OrihuelaEspina et al. (2010)) and hence for neurofeedback applications.

Recently, portable and wireless fNIRS devices have been developed (Pinti et al., 2018b) that can be used outside the laboratory. Such portable devices could be used to conduct neurofeedbacktraining studies at home, in school, or any other place in the world, including also low-resource countries (Pinti et al., 2018b). If the ease-of-use of these devices were to be further developed participants could even conduct neurofeedback training on their own and thus train whenever they want. This would make it easy to conduct neurofeedback studies with long training times at low costs. Of course, before moving out of the laboratory, standard protocols have to be developed and proven to be effective.

Also, fNIRS may be particularly suitable for interactive hyperscanning neurofeedback approaches, which have already been introduced by Duan et al., (2013), who had two participants competing in a "tug-of-war" neurofeedback game. This competitive context may increase motivation. In the future, cooperative approaches may be introduced as well, where two or more participants regulate a target brain signal together instead of competing. 
Recent developments in fMRI-neurofeedback protocols could be transferred to fNIRSneurofeedback. To date none of the fNIRS-neurofeedback studies used connectivity measures as a source of neurofeedback (other than support vector machine-based techniques). Connectivity-based neurofeedback has already been developed for fMRI-neurofeedback (Koush et al., 2015; Spetter et al., 2017; Yamashita et al., 2017; Zhao et al., 2019). Generally, some of these methods could be transferred to fNIRS since the sources and detectors can be freely positioned and might allow similar regions to those used in fMRI-neurofeedback studies to be covered. The high sampling of fNIRS allows stable correlations to be estimated in potentially shorter time windows, which is an advantage in this regard. However, these protocols should be established with caution and only with proper control of extracranial artifacts (e.g., using partial correlation and/or short-distance channels), which may easily produce spurious correlations. Future studies could also be oriented to recent methodological developments in fMRI-neurofeedback, such as implicit/covert neurofeedback protocols (i.e., neurofeedback without participants' awareness; e.g., Ramot et al., 2017) or decoded neurofeedback (Shibata et al., 2019)) and process-based neurofeedback, where protocols are designed to target disorder-specific processes (Lubianiker et al., 2019). Also, fMRI-informed approaches could be explored, as already applied in EEG-neurofeedback (e.g., Meir-Hasson et al., 2016), where fNIRS channels would predict the fMRI signal of a target region. Such approaches, if feasible with fNIRS, could result in improved spatial specificity and ideally make it possible to assess subcortical brain regions.

The problem of not reporting important information about signal-processing methods is also clearly visible in fMRI-neurofeedback studies and was addressed in a recent review by Heunis et al. (2020) recommending more rigorous reporting and development of methodological reporting standards. These recommendations can also be applied to fNIRS-neurofeedback research and similar default measures such as signal- or contrast-to-noise ratio calculations for evaluating fNIRS-signal quality could be established to make results more comparable across studies and to improve reproducibility (Heunis et al., 2020). Notably, fMRI-neurofeedback can already rely on a further developed field, and efforts with regard to standardization have already been made (Nichols et al., 2016). This is not the case for fNIRS research, where even standards for offline analysis methods are still lacking (Kamran et al., 2016; Pinti et al., 2018b). However, discussing this issue now will help to improve reporting quality and reproducibility at an early stage.

In general, the field will benefit from adopting more rigorous research and reporting practices as encouraged by a recent consensus (Aczel et al., 2019; Ros et al., 2020) to improve the likelihood of replicability and reproducibility (Mehler, 2019). These measures include sampling plans that are ideally based on adequate power analyses which render both positive and negative findings more informative (Mehler et al., 2019). We acknowledge that it remains challenging to define the smallest effect sizes of interest, which are context-specific. We thus recommend that researchers explore different approaches which have been established to define SESOIs (Lakens et al., 2018). We also acknowledge that neurofeedback studies are very resource-intensive requiring several training sessions and hence sampling plans that require relatively large sample sizes may be unrealistic to implement, particularly when working with patients. We therefore recommend considering ways of collaboration including multilab studies or multicenter trials and exploring alternatives such as sequential sampling methods with flexible stopping that can yield higher sensitivity (Schönbrodt and Wagenmakers, 2018) and encourage transparency in reporting sampling plans (e.g., mentioning practical constraints). Researchers can efficiently document design decisions, including the sampling and planned analyses, by publishing their protocols. These additional publishing formats include preregistration, where researchers document their methodology with a timestamp on a public platform such as the Open Science Framework before data acquisition starts (e.g., Mehler et al., 
2017). An alternative approach is to publish trial protocols in dedicated journals, which may be undertaken in parallel to data acquisition (e.g., Cox et al., 2016). Moreover, the recently introduced publishing format Registered Reports includes an initial peer-review stage that can grant authors acceptance in principle for their work independent of the statistical outcome. We note that adopting such methods is challenging: they involve additional costs such as more time in the preparation phase of the study and less flexibility (Allen and Mehler, 2019). However, recent preliminary meta-research suggests that the chances of publishing findings that do not meet traditional statistical thresholds increases remarkably for studies published as Registered Reports (Allen and Mehler, 2019; Scheel et al., 2020) while citation counts are comparable to traditional papers (Hummer et al., 2019). Hence, increased transparency not only benefits the field, but likely also individual authors. We therefore recommend that researchers should consider these publishing formats for future studies.

Lastly, it will be crucial to further develop standards and agreements, particularly for neurofeedback success measures, in order to have comparable outcome variables in the future (Haugg et al., 2020). We repeat suggestions by Paret et al., (2019) that a basic science approach should be employed, systematically exploring and optimizing neurofeedback protocols and real-time signal-processing methods, which can then inform translational work in the field.

\section{Conclusion}

The present systematic review of fNIRS-neurofeedback studies suggests, although tentatively, that people can regulate hemodynamic signals from different cortical brain regions with fNIRSneurofeedback indicating the feasibility of modulating normal behavior and psychiatric and neurological conditions. However, the field is at an early stage and consists mostly of feasibility, pilot, or proof-of-concept studies, so that the current systematic review might help to optimize future neurofeedback study designs but cannot provide recommendations on what neurofeedback targets, populations, and training protocols have proven most beneficial. There is room for improvement in reporting important information and statistical power, which impedes valid conclusions about specific behavioral effects or potential clinical utility of the method.

Nevertheless, fNIRS is becoming a viable method for neurofeedback research and has the potential for clinical translation of neurofeedback. Along this avenue, further methodological improvements, particularly aiming at improving signal quality, are of crucial importance and, together with more rigorous research and reporting practices, may improve the chances of replicability and reproducibility. This will help to gain a more solid understanding of fNIRS-neurofeedback and move the field closer towards agreements and standardization. FNIRS-neurofeedback is still in its infancy, and we now have the chance to create a solid foundation to build upon in the future. With this systematic review, we hope to stimulate a discussion about methodological and reporting standards at this early stage. 


\section{Data Availability Statement}

Data from this systematic review are available in the Supplementary material as well as at the Open Science Framework (see https://osf.io/hnxfq/), where we plan to update the database regularly in the future.

\section{Conflict of Interest}

Michael Lührs is an employee of the research company Brain Innovation B.V., Maastricht, The Netherlands. He was not involved in data extraction and quality assessments of the studies. He mainly contributed to the methodological section summarizing the current state of methods used for fNIRS real-time processing and discussing recent methodological developments in the field. Robert T. Thibault has received payments to consult with neurofeedback start-up companies.

All other authors have nothing to disclose.

\section{Author Contributions}

SK conceptualized the review with input from all other authors. SK selected the studies, extracted the data, conducted the analysis, assessed the quality of the studies, drafted and revised the manuscript. DM assessed the quality of the studies and revised the manuscript. ML revised the manuscript. RT revised the manuscript; KK revised the manuscript; BS supervised the drafting of the manuscript and revised it. All authors contributed to and approved the final version of the manuscript.

\section{Funding}

This work was supported by the International Research Training Group, The Neuroscience of Modulating Aggression and Impulsivity in Psychopathology (IRTG-2150) of the German Research Foundation (DFG). Robert T. Thibault is supported by a postdoctoral fellowship from the Fonds de la recherche en santé du Québec. Simon H. Kohl is supported by a fellowship from the Japan Society for the Promotion of Science (JSPS).

\section{Acknowledgments}

We would like to thank Johannes Fendel for providing valuable input on systematic review methods, Laura Bell for her help in creating the figures, and Jenny Le for preparatory work for data extraction. We also thank Franziska Klein for comments on an earlier draft of the current manuscript. Lastly, special thanks are due to all the authors of the included studies, who made this systematic review possible and provided feedback on the data extraction.

\section{References}

Aczel, B., Szaszi, B., Sarafoglou, A., Kekecs, Z., Kucharský, Š., Benjamin, D., et al. (2019). A consensus-based transparency checklist. Nat. Hum. Behav., 1-3. doi:10.1038/s41562-019-07726.

Algermissen, J., and Mehler, D. M. A. (2018). May the power be with you: are there highly powered studies in neuroscience, and how can we get more of them? J. Neurophysiol. 119, 2114-2117. doi:10.1152/jn.00765.2017.

Allen, C. P. G., and Mehler, D. M. A. (2019). Open science challenges, benefits and tips in early 
career and beyond. PLoS Biol. 17, e3000246. doi:10.1371/journal.pbio.3000246.

Aranyi, G., Pecune, F., Charles, F., Pelachaud, C., and Cavazza, M. (2016). Affective interaction with a virtual character through an fNIRS brain-computer interface. Front. Comput. Neurosci. 10. doi:10.3389/fncom.2016.00070.

Arifler, D., Zhu, T., Madaan, S., and Tachtsidis, I. (2015). Optimal wavelength combinations for near-infrared spectroscopic monitoring of changes in brain tissue hemoglobin and cytochrome $\mathrm{c}$ oxidase concentrations. Biomed. Opt. Express 6, 933. doi:10.1364/boe.6.000933.

Barnett, S. M., and Ceci, S. J. (2002). When and where do we apply what we learn? A taxonomy for far transfer. Psychol. Bull. 128, 612-637. doi:10.1037/0033-2909.128.4.612.

Barth, B., Strehl, U., Fallgatter, A. J., and Ehlis, A. C. (2016). Near-infrared spectroscopy based neurofeedback of prefrontal cortex activity: A proof-of-concept study. Front Hum Neurosci 10, 633. doi:10.3389/fnhum.2016.00633.

Birbaumer, N., Ruiz, S., and Sitaram, R. (2013). Learned regulation of brain metabolism. Trends Cogn. Sci. 17, 295-302. doi:10.1016/j.tics.2013.04.009.

Blume, F., Hudak, J., Dresler, T., Ehlis, A.-C., Kühnhausen, J., Renner, T. J., et al. (2017). NIRSbased neurofeedback training in a virtual reality classroom for children with attentiondeficit/hyperactivity disorder: study protocol for a randomized controlled trial. Trials 18, 41. doi:10.1186/s13063-016-1769-3.

Bluschke, A., Broschwitz, F., Kohl, S., Roessner, V., and Beste, C. (2016). The neuronal mechanisms underlying improvement of impulsivity in ADHD by theta/beta neurofeedback. Sci. Rep. 6, 31178. doi:10.1038/srep31178.

Brigadoi, S., and Cooper, R. J. (2015). How short is short? Optimum source-detector distance for short-separation channels in functional near-infrared spectroscopy. Neurophotonics 2, 025005. doi:10.1117/1.NPh.2.2.025005.

Button, K. S., Ioannidis, J. P. A., Mokrysz, C., Nosek, B. A., Flint, J., Robinson, E. S. J., et al. (2013). Power failure: why small sample size undermines the reliability of neuroscience. Nat. Rev. Neurosci. 14, 365-376. doi:10.1038/nrn3475.

Calamia, M., Markon, K., and Tranel, D. (2013). The Robust Reliability of Neuropsychological Measures : Meta- Analyses of Test - Retest Correlations. Clin. Neuropsychol. 27, 1077-1105. doi:10.1080/13854046.2013.809795.

Caldwell, M., Scholkmann, F., Wolf, U., Wolf, M., Elwell, C., and Tachtsidis, I. (2016). Modelling confounding effects from extracerebral contamination and systemic factors on functional nearinfrared spectroscopy. Neuroimage 143, 91-105. doi:10.1016/j.neuroimage.2016.08.058.

Cohen, A., Keynan, J. N., Jackont, G., Green, N., Rashap, I., Shani, O., et al. (2016). Multi-modal Virtual Scenario Enhances Neurofeedback Learning. Front. Robot. AI 3, 52. doi:10.3389/frobt.2016.00052.

Cohen, J. (1992). A Power Primer. Psychol. Bull. 112, 155-159. doi:10.1038/141613a0.

Cox, W. M., Subramanian, L., Linden, D. E. J., Lührs, M., McNamara, R., Playle, R., et al. (2016). Neurofeedback training for alcohol dependence versus treatment as usual: study protocol for a randomized controlled trial. Trials 17, 480. doi:10.1186/s13063-016-1607-7.

Cui, X., Bray, S., Bryant, D. M., Glover, G. H., and Reiss, A. L. (2011). A quantitative comparison of NIRS and fMRI across multiple cognitive tasks. Neuroimage 54, 2808-21. 
fNIRS-Neurofeedback - a systematic review

doi:10.1016/j.neuroimage.2010.10.069.

Cui, X., Bray, S., and Reiss, A. L. (2010). Functional near infrared spectroscopy (NIRS) signal improvement based on negative correlation between oxygenated and deoxygenated hemoglobin dynamics. Neuroimage 49, 3039-3046. doi:10.1016/j.neuroimage.2009.11.050.

Duan, L., Liu, W. J., Dai, R. N., Li, R., Lu, C. M., Huang, Y. X., et al. (2013). Cross-Brain Neurofeedback: Scientific Concept and Experimental Platform. PLoS One 8, 1-5. doi:10.1371/journal.pone.0064590.

Ehlis, A. C., Barth, B., Hudak, J., Storchak, H., Weber, L., Kimmig, A. C. S., et al. (2018). NearInfrared Spectroscopy as a New Tool for Neurofeedback Training: Applications in Psychiatry and Methodological Considerations. Jpn. Psychol. Res. 60, 225-241. doi:10.1111/jpr.12225.

Emmert, K., Kopel, R., Sulzer, J., Brühl, A. B., Berman, B. D., Linden, D. E. J., et al. (2016). Metaanalysis of real-time fMRI neurofeedback studies using individual participant data: How is brain regulation mediated? Neuroimage 124, 806-812. doi:10.1016/j.neuroimage.2015.09.042.

Engel, L., Beaton, D. E., and Touma, Z. (2018). Minimal Clinically Important Difference: A Review of Outcome Measure Score Interpretation. Rheum. Dis. Clin. North Am. 44, 177-188. doi:10.1016/j.rdc.2018.01.011.

Faul, F., Erdfelder, E., Lang, A. G., and Buchner, A. (2007). G*Power 3: A flexible statistical power analysis program for the social, behavioral, and biomedical sciences. Behav. Res. Methods 39, 175-191. doi:10.3758/BF03193146.

Ferrari, M., and Quaresima, V. (2012). A brief review on the history of human functional nearinfrared spectroscopy (fNIRS) development and fields of application. Neuroimage 63, 921-935. doi:10.1016/j.neuroimage.2012.03.049.

Fujimoto, H., Mihara, M., Hattori, N., Hatakenaka, M., Yagura, H., Kawano, T., et al. (2017). Neurofeedback-induced facilitation of the supplementary motor area affects postural stability. Neurophotonics 4. doi:10.1117/1.NPh.4.4.045003.

Gelman, A. (2018, March 15). You need 16 times the sample size to estimate an interaction than to estimate a main effect [Blog post]. Retrieved from https://statmodeling.stat.columbia.edu/2018/03/15/need-16-times-sample-size-estimateinteraction-estimate-main-effect/\#comment-685111/

Goldway, N., Ablin, J., Lubin, O., Zamir, Y., Keynan, J. N., Or-Borichev, A., et al. (2019). Volitional limbic neuromodulation exerts a beneficial clinical effect on Fibromyalgia. Neuroimage 186, 758-770. doi:10.1016/j.neuroimage.2018.11.001.

Gruzelier, J., Inoue, A., Smart, R., Steed, A., and Steffert, T. (2010). Acting performance and flow state enhanced with sensory-motor rhythm neurofeedback comparing ecologically valid immersive VR and training screen scenarios. Neurosci. Lett. 480, 112-6. doi:10.1016/j.neulet.2010.06.019.

Hatakenaka, M., Miyai, I., Mihara, M., Sakoda, S., and Kubota, K. (2007). Frontal regions involved in learning of motor skill—A functional NIRS study. Neuroimage 34, 109-116. doi:10.1016/J.NEUROIMAGE.2006.08.014.

Haugg, A., Sladky, R., Skouras, S., McDonald, A., Craddock, C., Kirschner, M., et al. (2020). Can we predict real-time fMRI neurofeedback learning success from pre-training brain activity? bioRxiv, 2020.01.15.906388. doi:10.1101/2020.01.15.906388.

He, K., Liang, Y., Abdollahi, F., Fisher Bittmann, M., Kording, K., and Wei, K. (2016). The 
Statistical Determinants of the Speed of Motor Learning. PLOS Comput. Biol. 12, e1005023. doi:10.1371/journal.pcbi.1005023.

Heunis, S., Lamerichs, R., Zinger, S., Caballero-Gaudes, C., Jansen, J. F. A., Aldenkamp, B., et al. (2020). Quality and denoising in real-time functional magnetic resonance imaging neurofeedback: A methods review. Hum. Brain Mapp. doi:10.1002/hbm.25010.

Hinterberger, T., Neumann, N., Pham, M., Kübler, A., Grether, A., Hofmayer, N., et al. (2004). A multimodal brain-based feedback and communication system. Exp. Brain Res. 154, 521-6. doi:10.1007/s00221-003-1690-3.

Hosseini, S. M. H. H., Pritchard-Berman, M., Sosa, N., Ceja, A., and Kesler, S. R. (2016). Taskbased neurofeedback training: A novel approach toward training executive functions. Neuroimage 134, 153-159. doi:10.1016/j.neuroimage.2016.03.035.

Hudak, J., Blume, F., Dresler, T., Haeussinger, F. B., Renner, T. J., Fallgatter, A. J., et al. (2017a). Near-infrared spectroscopy-based frontal lobe neurofeedback integrated in virtual reality modulates brain and behavior in highly impulsive adults. Front Hum Neurosci 11. doi:10.3389/fnhum.2017.00425.

Hudak, J., Blume, F., Dresler, T., Haeussinger, F. B., Renner, T. J., Fallgatter, A. J., et al. (2017b). Near-Infrared Spectroscopy-Based Frontal Lobe Neurofeedback Integrated in Virtual Reality Modulates Brain and Behavior in Highly Impulsive Adults. Front. Hum. Neurosci. 11, 1-13. doi:10.3389/fnhum.2017.00425.

Hudak, J., Rosenbaum, D., Barth, B., Fallgatter, A. J., and Ehlis, A. C. (2018). Functionally disconnected: A look at how study design influences neurofeedback data and mechanisms in attention-deficit/hyperactivity disorder. PLoS One 13, 1-15. doi:10.1371/journal.pone.0200931.

Hummer, L. T., Singleton Thorn, F., Nosek, B. A., and Errington, T. M. (2019). Evaluating Registered Reports: A Naturalistic Comparative Study of Article Impact Short. PsyArXiv, 1-34. doi:10.31219/osf.io/5y8w7.

Ioannidis, J. P. A. (2008). Why most discovered true associations are inflated. Epidemiology 19, 640-648. doi:10.1097/EDE.0b013e31818131e7.

Jasper, H. H. (1958). The Ten-Twenty Electrode System of the International Federation. Electroencephalogr. Clin. Neurophysiol. 10, 371-375. Available at: https://ci.nii.ac.jp/naid/10017996828/ [Accessed November 8, 2019].

Kanoh, S., Susila, I., and Miyamoto, K. (2011). The Effect of Neurofeedback Training on Cortical Activity during Motor Imagery Revealed by NIRS and fMRI. Int. J. Bioelectromagn. 13, 82-83. Available at: http://133.25.196.106/2006-/volume13/number2/2011_v13_no2_82-83.pdf.

Kamran, M. A., Mannan, M. M. N., and Jeong, M. Y. (2016). Cortical signal analysis and advances in functional near-infrared spectroscopy signal: A review. Front Hum Neurosci 10. doi:10.3389/fnhum.2016.00261.

Kimmig, A.-C. S. C. S., Dresler, T., Hudak, J., Haeussinger, F. B., Wildgruber, D., Fallgatter, A. J., et al. (2018). Feasibility of NIRS-based neurofeedback training in social anxiety disorder: behavioral and neural correlates. J. Neural Transm. 0, 0. doi:10.1007/s00702-018-1954-5.

Kinoshita, A., Takizawa, R., Yahata, N., Homae, F., Hashimoto, R., Sakakibara, E., et al. (2016). Development of a neurofeedback protocol targeting the frontal pole using near-infrared spectroscopy. Psychiatry Clin Neurosci 70, 507-516. doi:10.1111/pcn.12427.

Kirilina, E., Jelzow, A., Heine, A., Niessing, M., Wabnitz, H., Brühl, R., et al. (2012). The 
physiological origin of task-evoked systemic artefacts in functional near infrared spectroscopy. Neuroimage 61, 70-81. doi:10.1016/J.NEUROIMAGE.2012.02.074.

Klein, F., and Kranczioch, C. (2019). Signal Processing in fNIRS: A Case for the Removal of Systemic Activity for Single Trial Data. Front. Hum. Neurosci. 13, 331.

doi:10.3389/fnhum.2019.00331.

Kober, S. E., Gressenberger, B., Kurzmann, J., Neuper, C., and Wood, G. (2015). Voluntary modulation of hemodynamic responses in swallowing related motor areas: A near-infrared spectroscopy-based neurofeedback study. PLoS One 10, 1-17.

doi:10.1371/journal.pone.0143314.

Kober, S. E., Hinterleitner, V., Bauernfeind, G., Neuper, C., and Wood, G. (2018). Trainability of hemodynamic parameters: A near-infrared spectroscopy based neurofeedback study. Biol Psychol 136, 168-180. doi:10.1016/j.biopsycho.2018.05.009.

Kober, S. E., Wood, G., Kurzmann, J., Friedrich, E. V. C., Stangl, M., Wippel, T., et al. (2014). Near-infrared spectroscopy based neurofeedback training increases specific motor imagery related cortical activation compared to sham feedback. Biol. Psychol. 95, 21-30. doi:10.1016/j.biopsycho.2013.05.005.

Kohl, S. H., Veit, R., Spetter, M. S., Günther, A., Rina, A., Lührs, M., et al. (2019). Real-time fMRI neurofeedback training to improve eating behavior by self-regulation of the dorsolateral prefrontal cortex: A randomized controlled trial in overweight and obese subjects. Neuroimage 191, 596-609. doi:10.1016/J.NEUROIMAGE.2019.02.033.

Koush, Y., Meskaldji, D.-E., Pichon, S., Rey, G., Rieger, S. W., Linden, D. E. J., et al. (2015). Learning control over emotion networks through connectivity-based neurofeedback. Cereb. Cortex, bhv311. Available at: https://academic.oup.com/cercor/article/27/2/1193/3056240/Learning-Control-Over-EmotionNetworks-Through.

Lakens, D., Scheel, A. M., and Isager, P. (2018). Equivalence testing for psychological research: A tutorial. Adv. Methods Pract. Psychol. Sci., 1-11. doi:10.17605/OSF.IO/V3ZKT.

Lapborisuth, P., Zhang, X., Noah, A., and Hirsch, J. (2017). Neurofeedback-based functional nearinfrared spectroscopy upregulates motor cortex activity in imagined motor tasks.

Neurophotonics 4, 021107. doi:10.1117/1.NPh.4.2.021107.

Lee, S. H., Jang, G. H., Jin, S. H., and An, J. (2015). Neuro-feedback using real-time near infrared spectroscopy enhances brain plasticity during treadmill walking: A pilot study. in IEEE/ASME International Conference on Advanced Intelligent Mechatronics, AIM, 437-440. doi:10.1109/AIM.2015.7222572.

Li, K., Kendrick, K. M., and Becker, B. (2019). Functional near-infrared spectroscopy-informed neurofeedback : regional-specific modulation of lateral orbitofrontal activation and cognitive flexibility. 6. doi:10.1117/1.NPh.6.2.025011.

Liu, N., Cliffer, S., Pradhan, A. H., Lightbody, A., Hall, S. S., and Reiss, A. L. (2016). Opticalimaging-based neurofeedback to enhance therapeutic intervention in adolescents with autism: methodology and initial data. Neurophotonics 4, 011003. doi:10.1117/1.NPh.4.1.011003.

Lloyd-Fox, S., Blasi, A., and Elwell, C. E. (2010). Illuminating the developing brain: The past, present and future of functional near infrared spectroscopy. Neurosci. Biobehav. Rev. 34, 269284. doi:10.1016/j.neubiorev.2009.07.008. 
Lubianiker, N., Goldway, N., Fruchtman-Steinbok, T., Paret, C., Keynan, J. N., Singer, N., et al. (2019). Process-based framework for precise neuromodulation. Nat. Hum. Behav. 3, 436-445. doi:10.1038/s41562-019-0573-y.

Marx, A.-M. M., Ehlis, A.-C. C., Furdea, A., Holtmann, M., Banaschewski, T., Brandeis, D., et al. (2015). Near-infrared spectroscopy (NIRS) neurofeedback as a treatment for children with attention deficit hyperactivity disorder (ADHD)-a pilot study. Front Hum Neurosci 8, 1038. doi:10.3389/fnhum.2014.01038.

Mathiak, K. A., Alawi, E. M., Koush, Y., Dyck, M., Cordes, J. S., Gaber, T. J., et al. (2015). Social reward improves the voluntary control over localized brain activity in fMRI-based neurofeedback training. Front. Behav. Neurosci. 9, 136. doi:10.3389/fnbeh.2015.00136.

Mayer, K., Blume, F., Wyckoff, S. N., Brokmeier, L. L., and Strehl, U. (2016). Neurofeedback of slow cortical potentials as a treatment for adults with Attention Deficit-/Hyperactivity Disorder. Clin. Neurophysiol. 127, 1374-1386. doi:10.1016/J.CLINPH.2015.11.013.

Mayer, K., Wyckoff, S. N., Fallgatter, A. J., Ehlis, A.-C. C., and Strehl, U. (2015). Neurofeedback as a nonpharmacological treatment for adults with attention-deficit/hyperactivity disorder (ADHD): study protocol for a randomized controlled trial. Trials 16, 174. doi:10.1186/s13063015-0683-4.

Mehler, D. (2019). “The replication challenge: Is brain imaging next?," in Casting Light on the Dark Side of Brain Imaging, eds. A. Raz and R. T. Thibault (Academic Press), 84-88. doi:10.1016/B978-0-12-816179-1.00010-4.

Mehler, D. M. A., Edelsbrunner, P. A., and Matić, K. (2019). Appreciating the Significance of Nonsignificant Findings in Psychology. J. Eur. Psychol. Students 10, 1-7. doi:10.5334/jeps.e2019a.

Mehler, D. M. A., Williams, A. N., Whittaker, J. R., Krause, F., Lührs, M., Wise, R. G., et al. (2017). Study Pre-registration: Gradual real-time fMRI neurofeedback training of motor imagery in middle cerebral artery stroke patients. Available at: https://osf.io/7q68p/ [Accessed April 22, 2020].

Meir-Hasson, Y., Keynan, J. N., Kinreich, S., Jackont, G., Cohen, A., Podlipsky-Klovatch, I., et al. (2016). One-Class FMRI-Inspired EEG Model for Self-Regulation Training. PLoS One 11, e0154968. doi:10.1371/journal.pone.0154968.

Mihara, M., Hattori, N., Hatakenaka, M., Yagura, H., Kawano, T., Hino, T., et al. (2013). Nearinfrared spectroscopy-mediated neurofeedback enhances efficacy of motor imagery-based training in poststroke victims: A pilot study. Stroke 44, 1091-1098.

doi:10.1161/STROKEAHA.111.674507.

Mihara, M., Miyai, I., Hattori, N., Hatakenaka, M., Yagura, H., Kawano, T., et al. (2012). Neurofeedback using real-time near-infrared spectroscopy enhances motor imagery related cortical activation. PLoS One 7, e32234. doi:10.1371/journal.pone.0032234.

Moher, D., Liberati, A., Tetzlaff, J., Altman, D. G., and Group, T. P. (2009). Preferred Reporting Items for Systematic Reviews and Meta-Analyses: The PRISMA Statement. PLoS Med. 6, e1000097. doi:10.1371/journal.pmed.1000097.

Narita, N. (2015). Application of NIRS as a non-invasive and supportive tool for autism spectrum disorders. Trans. Japanese Soc. Med. Biol. Eng. 53, S153_01-S153_01. doi:10.11239/jsmbe.53.S153_01.

Naseer, N., and Hong, K.-S. S. (2015). fNIRS-based brain-computer interfaces: a review. Front Hum 
fNIRS-Neurofeedback - a systematic review

Neurosci 9, 3. doi:10.3389/fnhum.2015.00003.

Nichols, T. E., Das, S., Eickhoff, S. B., Evans, A. C., Glatard, T., Hanke, M., et al. (2016). Best Practices in Data Analysis and Sharing in Neuroimaging using MRI. bioRxiv, 054262. doi:10.1101/054262.

Nieuwenhuis, S., Forstmann, B. U., and Wagenmakers, E. J. (2011). Erroneous analyses of interactions in neuroscience: A problem of significance. Nat. Neurosci. 14, 1105-1107. doi:10.1038/nn.2886.

Ninaus, M., Kober, S. E., Witte, M., Koschutnig, K., Stangl, M., Neuper, C., et al. (2013). Neural substrates of cognitive control under the belief of getting neurofeedback training. Front. Hum. Neurosci. 7, 914. doi:10.3389/fnhum.2013.00914.

Ogawa, S., Tank, D. W., Menon, R., Ellermann, J. M., Kim, S. G., Merkle, H., et al. (1992). Intrinsic signal changes accompanying sensory stimulation: Functional brain mapping with magnetic resonance imaging. Proc. Natl. Acad. Sci. U. S. A. 89, 5951-5955. doi:10.1073/pnas.89.13.5951.

Okamoto, M., Dan, H., Sakamoto, K., Takeo, K., Shimizu, K., Kohno, S., et al. (2004). Threedimensional probabilistic anatomical cranio-cerebral correlation via the international 10-20 system oriented for transcranial functional brain mapping. Neuroimage 21, 99-111. doi:10.1016/J.NEUROIMAGE.2003.08.026.

Orihuela-Espina, F., Leff, D. R., James, D. R. C., Darzi, A. W., and Yang, G. Z. (2010). Quality control and assurance in functional near infrared spectroscopy (fNIRS) experimentation. Phys. Med. Biol. 55, 3701-3724. doi:10.1088/0031-9155/55/13/009.

Paret, C., Goldway, N., Zich, C., Keynan, J. N., Hendler, T., Linden, D., et al. (2019). Current progress in real-time functional magnetic resonance-based neurofeedback: methodological challenges and achievements. Academic Press doi:10.1016/J.NEUROIMAGE.2019.116107.

Pfeifer, M. D., Scholkmann, F., and Labruyère, R. (2018). Signal Processing in Functional NearInfrared Spectroscopy ( fNIRS ): Methodological Differences Lead to Different Statistical Results. 11, 1-12. doi:10.3389/fnhum.2017.00641.

Pinti, P., Aichelburg, C., Gilbert, S., Hamilton, A., Hirsch, J., Burgess, P., et al. (2018a). A Review on the Use of Wearable Functional Near-Infrared Spectroscopy in Naturalistic Environments. Jpn. Psychol. Res. 60, 347-373. doi:10.1111/jpr.12206.

Pinti, P., Tachtsidis, I., Hamilton, A., Hirsch, J., Aichelburg, C., Gilbert, S., et al. (2018b). The present and future use of functional near-infrared spectroscopy (fNIRS) for cognitive neuroscience. Ann. N. Y. Acad. Sci., 1-25. doi:10.1111/nyas.13948.

Poldrack, R. A., Baker, C. I., Durnez, J., Gorgolewski, K. J., Matthews, P. M., Munafò, M. R., et al. (2017). Scanning the horizon: towards transparent and reproducible neuroimaging research. Nat. Rev. Neurosci. 18, 115-126. doi:10.1038/nrn.2016.167.

Poldrack, R. A., Paré-Blagoev, E. J., and Grant, P. E. (2002). Pediatric Functional Magnetic Resonance Imaging: Progress and Challenges. Top. Magn. Reson. Imaging 13, 61-70. doi:10.1097/00002142-200202000-00005.

Quaresima, and Ferrari (2019). A Mini-Review on Functional Near-Infrared Spectroscopy (fNIRS): Where Do We Stand, and Where Should We Go? Photonics 6, 87. doi:10.3390/photonics6030087.

Ramot, M., Kimmich, S., Gonzalez-Castillo, J., Roopchansingh, V., Popal, H., White, E., et al. (2017). Direct modulation of aberrant brain network connectivity through real-time 
NeuroFeedback. Elife 6. doi:10.7554/eLife.28974.

Rance, M., Walsh, C., Sukhodolsky, D. G., Pittman, B., Qiu, M., Kichuk, S. A., et al. (2018). Time course of clinical change following neurofeedback. Neuroimage 181, 807-813. doi:10.1016/j.neuroimage.2018.05.001.

Ros, T., Enriquez-Geppert, S., Zotev, V., Young, K. D., Wood, G., Whitfield-Gabrieli, S., et al. (2020). Consensus on the reporting and experimental design of clinical and cognitivebehavioural neurofeedback studies (CRED-nf checklist). Brain. doi:10.1093/brain/awaa009.

Sato, T., Nambu, I., Takeda, K., Aihara, T., Yamashita, O., Isogaya, Y., et al. (2016). Reduction of global interference of scalp-hemodynamics in functional near-infrared spectroscopy using short distance probes. Neuroimage 141, 120-132. doi:10.1016/j.neuroimage.2016.06.054.

Schabus, M., Griessenberger, H., Gnjezda, M.-T., Heib, D. P. J., Wislowska, M., and Hoedlmoser, K. (2017). Better than sham? A double-blind placebo-controlled neurofeedback study in primary insomnia. Brain 140, 1041-1052. doi:10.1093/brain/awx011.

Schäfer, T., and Schwarz, M. A. (2019). The Meaningfulness of Effect Sizes in Psychological Research : Differences Between Sub-Disciplines and the Impact of Potential Biases. Front. Psychol. 10. doi:10.3389/fpsyg.2019.00813.

Scharnowski, F., Veit, R., Zopf, R., Studer, P., Bock, S., Diedrichsen, J., et al. (2015). Manipulating motor performance and memory through real-time fMRI neurofeedback. Biol. Psychol. 108, 8597. doi:10.1016/j.biopsycho.2015.03.009.

Scheel, A. M., Schijen, M., and Lakens, D. (2020). An excess of positive results : Comparing the standard psychology literature with registered reports. 1-14. doi:10.31234/OSF.IO/P6E9C.

Scholkmann, F., Kleiser, S., Metz, A. J., Zimmermann, R., Mata Pavia, J., Wolf, U., et al. (2014). A review on continuous wave functional near-infrared spectroscopy and imaging instrumentation and methodology. Neuroimage 85, 6-27. doi:10.1016/j.neuroimage.2013.05.004.

Schönbrodt, F. D., and Wagenmakers, E. J. (2018). Bayes factor design analysis: Planning for compelling evidence. Psychon. Bull. Rev. 25, 128-142. doi:10.3758/s13423-017-1230-y.

Schönenberg, M., Wiedemann, E., Schneidt, A., Scheeff, J., Logemann, A., Keune, P. M., et al. (2017). Neurofeedback, sham neurofeedback, and cognitive-behavioural group therapy in adults with attention-deficit hyperactivity disorder: a triple-blind, randomised, controlled trial. The lancet. Psychiatry 4, 673-684. doi:10.1016/S2215-0366(17)30291-2.

Schulz, K. F., Altman, D. G., Moher, D., and CONSORT Group (2010). CONSORT 2010 statement: updated guidelines for reporting parallel group randomised trials. BMJ 340, c332. doi:10.1136/bmj.c332.

Sepulveda, P., Sitaram, R., Rana, M., Montalba, C., Tejos, C., and Ruiz, S. (2016). How Feedback, Motor Imagery, and Reward Influence Brain Self-Regulation Using Real-Time fMRI. Hum. Brain Mapp. 37, 3153-3171. doi:10.1002/hbm.23228.

Sherlin, L. H., Arns, M., Lubar, J., Heinrich, H., Kerson, C., Strehl, U., et al. (2011). Neurofeedback and Basic Learning Theory: Implications for Research and Practice. J. Neurother. 15, 292-304. doi:10.1080/10874208.2011.623089.

Shibata, K., Lisi, G., Cortese, A., Watanabe, T., Sasaki, Y., and Kawato, M. (2019). Toward a comprehensive understanding of the neural mechanisms of decoded neurofeedback. Neuroimage 188, 539-556. doi:10.1016/J.NEUROIMAGE.2018.12.022. 
Simmons, J. P., Nelson, L. D., and Simonsohn, U. (2011). False-Positive Psychology. Psychol. Sci. 22, 1359-1366. doi:10.1177/0956797611417632.

Singh, A. K., Okamoto, M., Dan, H., Jurcak, V., and Dan, I. (2005). Spatial registration of multichannel multi-subject fNIRS data to MNI space without MRI. Neuroimage 27, 842-851. doi:10.1016/J.NEUROIMAGE.2005.05.019.

Sitaram, R., Ros, T., Stoeckel, L., Haller, S., Scharnowski, F., Lewis-Peacock, J., et al. (2017). Closed-loop brain training: the science of neurofeedback. Nat. Rev. Neurosci. 18, 86-100. doi:10.1038/nrn.2016.164.

Skinner, B. F. (1963). Operant behavior. Am. Psychol. 18, 503-515. doi:10.1037/h0045185.

Sorger, B., Scharnowski, F., Linden, D. E. J., Hampson, M., and Young, K. D. (2019). Control freaks: Towards optimal selection of control conditions for fMRI neurofeedback studies. Neuroimage 186, 256-265. doi:10.1016/j.neuroimage.2018.11.004.

Spetter, M. S., Malekshahi, R., Birbaumer, N., Lührs, M., van der Veer, A. H., Scheffler, K., et al. (2017). Volitional regulation of brain responses to food stimuli in overweight and obese subjects: A real-time fMRI feedback study. Appetite 112, 188-195. doi:10.1016/j.appet.2017.01.032.

Stangl, M., Bauernfeind, G., Kurzmann, J., Scherer, R., and Neuper, C. (2013). A haemodynamic brain-computer interface based on real-time classification of near infrared spectroscopy signals during motor imagery and mental arithmetic. J. Near Infrared Spectrosc. 21, 157-171. doi:10.1255/jnirs.1048.

Stone, W. L., and Lemanek, K. L. (1990). "Developmental issues in children's self-reports.," in Through the eyes of the child: Obtaining self-reports from children and adolescents, ed. A. M. La Greca (Allyn \& Bacon), 18-56.

Storchak, H., Hudak, J., Haeussinger, F. B., Rosenbaum, D., Fallgatter, A. J., and Ehlis, A. C. (2018). Reducing auditory verbal hallucinations by means of fNIRS neurofeedback - A case study with a paranoid schizophrenic patient. Schizophr. Res., 41-43. doi:10.1016/j.schres.2018.09.018.

Strehl, U., Aggensteiner, P., Wachtlin, D., Brandeis, D., Albrecht, B., Arana, M., et al. (2017). Neurofeedback of Slow Cortical Potentials in Children with Attention-Deficit/Hyperactivity Disorder: A Multicenter Randomized Trial Controlling for Unspecific Effects. Front. Hum. Neurosci. 11, 135. doi:10.3389/fnhum.2017.00135.

Szucs, D., and Ioannidis, J. P. A. A. (2017). Empirical assessment of published effect sizes and power in the recent cognitive neuroscience and psychology literature. 15, e2000797. doi:10.1371/journal.pbio.2000797.

Tachtsidis, I., and Scholkmann, F. (2016). False positives and false negatives in functional nearinfrared spectroscopy: issues, challenges, and the way forward. Neurophotonics 3, 031405. doi:10.1117/1.nph.3.3.031405.

Thibault, R. T., Lifshitz, M., and Raz, A. (2016). The self-regulating brain and neurofeedback: Experimental science and clinical promise. Cortex 74, 247-261. doi:10.1016/j.cortex.2015.10.024.

Thibault, R. T., MacPherson, A., Lifshitz, M., Roth, R. R., and Raz, A. (2018). Neurofeedback with fMRI: A critical systematic review. Neuroimage 172, 786-807. doi:10.1016/j.neuroimage.2017.12.071.

Thibault, R. T., and Raz, A. (2016). Neurofeedback: the power of psychosocial therapeutics. The 
fNIRS-Neurofeedback - a systematic review

Lancet Psychiatry 3, e18. Available at:

http://www.thelancet.com/journals/lanpsy/article/PIIS2215-0366(16)30326-1/abstract.

Trambaiolli, L. R., Biazoli, C. E., Cravo, A. M., Falk, T. H., and Sato, J. R. (2018). Functional nearinfrared spectroscopy-based affective neurofeedback: feedback effect, illiteracy phenomena, and whole-connectivity profiles. Neurophotonics 5, 1. doi:10.1117/1.nph.5.3.035009.

Tsuzuki, D., and Dan, I. (2014). Spatial registration for functional near-infrared spectroscopy: From channel position on the scalp to cortical location in individual and group analyses. Neuroimage 85, 92-103. doi:10.1016/J.NEUROIMAGE.2013.07.025.

Tsuzuki, D., Jurcak, V., Singh, A. K., Okamoto, M., Watanabe, E., and Dan, I. (2007). Virtual spatial registration of stand-alone fNIRS data to MNI space. Neuroimage 34, 1506-1518. doi:10.1016/j.neuroimage.2006.10.043.

Tufanaru, C., Munn, Z., Aromataris, E., Campbell, J., and Hopp, L. (2017). “Chapter 3: Systematic reviews of effectiveness.," in Joanna Briggs Institute Reviewer's Manual., eds. E. Aromataris and Z. Munn (The Joanna Briggs Institute). Available at:

https://reviewersmanual.joannabriggs.org/.

Van Doren, J., Arns, M., Heinrich, H., Vollebregt, M. A., Strehl, U., and K. Loo, S. (2019). Sustained effects of neurofeedback in ADHD: a systematic review and meta-analysis. Eur. Child Adolesc. Psychiatry 28, 293-305. doi:10.1007/s00787-018-1121-4.

Veenman, M. V. J. (2011). Alternative assessment of strategy use with self-report instruments: A discussion. Metacognition Learn. 6, 205-211. doi:10.1007/s11409-011-9080-x.

von Lühmann, A., Herff, C., Heger, D., and Schultz, T. (2015). Toward a wireless open source instrument: Functional near-infrared spectroscopy in mobile neuroergonomics and BCI applications. Front. Hum. Neurosci. 9, 617. doi:10.3389/fnhum.2015.00617.

von Lühmann, A., Ortega-Martinez, A., Boas, D. A., and Yücel, M. A. (2020). Using the General Linear Model to Improve Performance in fNIRS Single Trial Analysis and Classification: A Perspective. Front. Hum. Neurosci. 14, 30. doi:10.3389/fnhum.2020.00030.

Weyand, S., Takehara-Nishiuchi, K., and Chau, T. (2015). Weaning Off Mental Tasks to Achieve Voluntary Self-Regulatory Control of a Near-Infrared Spectroscopy Brain-Computer Interface. IEEE Trans. Neural Syst. Rehabil. Eng. 23, 548-561. doi:10.1109/TNSRE.2015.2399392.

Yamashita, A., Hayasaka, S., Kawato, M., and Imamizu, H. (2017). Connectivity Neurofeedback Training Can Differentially Change Functional Connectivity and Cognitive Performance. Cereb. Cortex 27, 4960-4970. doi:10.1093/cercor/bhx177.

Zhang, X., Noah, J. A., and Hirsch, J. (2016). Separation of the global and local components in functional near-infrared spectroscopy signals using principal component spatial filtering. Neurophotonics 3, 015004. doi:10.1117/1.NPh.3.1.015004.

Zhao, Z., Yao, S., Li, K., Sindermann, C., Zhou, F., Zhao, W., et al. (2019). Real-Time Functional Connectivity-Informed Neurofeedback of Amygdala-Frontal Pathways Reduces Anxiety. Psychother. Psychosom. 88, 5-15. doi:10.1159/000496057. 